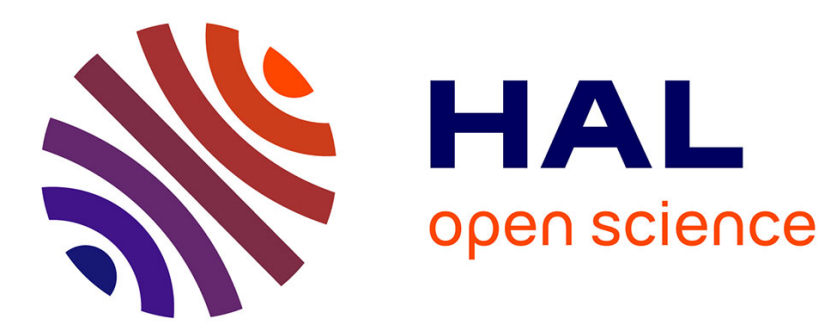

\title{
Un atelier de potiers du Ier siècle de notre ère à Mandelieu (A.-M.) \\ Lucien Rivet
}

\section{To cite this version:}

Lucien Rivet. Un atelier de potiers du Ier siècle de notre ère à Mandelieu (A.-M.). Documents d'archéologie méridionale, 1986, 9 (1), pp.119 - 134. 10.3406/dam.1986.975 . halshs-01744117

\section{HAL Id: halshs-01744117 \\ https://shs.hal.science/halshs-01744117}

Submitted on 16 Apr 2018

HAL is a multi-disciplinary open access archive for the deposit and dissemination of scientific research documents, whether they are published or not. The documents may come from teaching and research institutions in France or abroad, or from public or private research centers.
L'archive ouverte pluridisciplinaire HAL, est destinée au dépôt et à la diffusion de documents scientifiques de niveau recherche, publiés ou non, émanant des établissements d'enseignement et de recherche français ou étrangers, des laboratoires publics ou privés.

\section{(1)(1) $\$(0)$}

Distributed under a Creative Commons Attribution - NonCommercial - ShareAlikel 4.0 


\section{Un atelier de potiers du ler siècle de notre ère à Mandelieu (A.-}

M.)

\section{Lucien Rivet}

\section{Abstract}

A pottery workshop of the first cent. A.D. at Mandelieu (A.-M.).

An excavation of urgency conducted on the site of a roman villa in Alpes-Maritimes enabled the discovery of numerous discarted pottery workshop vases. The analysis of the context and the natural environment show a local production. Four types of articles are defined : common pottery made of clear or dark clay, small amphoras, tiles. This pottery workshop must have been intented essentially for the making of small amphoras, for the economical needs of the villa (or several ?) . It was in production during a brief laps of time that can be situated about the middle of the first cent. AD.

\section{Résumé}

Une fouille d'urgence menée sur une villa romaine des Alpes-Maritimes a permis, entre autres, la découverte de nombreux surcuits céramiques. L'analyse du contexte et du milieu naturel permettent de concevoir une production locale. Quatre catégories de fabrication sont définies (céramiques communes à pâte claire ou sombre ; petites amphores; tuiles). Cet atelier devait être destiné pour l'essentiel à la confection des petites amphores répondant aux besoins économiques de la villa (ou de plusieurs ?) ; il a produit durant un bref laps de temps que l'on situera vers le milieu du ler s. de n. è.

\section{Citer ce document / Cite this document :}

Rivet Lucien. Un atelier de potiers du ler siècle de notre ère à Mandelieu (A.-M.). In: Documents d'Archéologie Méridionale, vol. 9, 1986. pp. 119-134;

doi : 10.3406/dam.1986.975

http://www.persee.fr/doc/dam_0184-1068_1986_num_9_1_975

Document généré le 31/01/2018 


\title{
Un atelier de potiers du Ier siècle de notre ère à Mandelieu (A.-M.)
}

\author{
par Lucien RIVET *
}

Résumé - Une fouille d'urgence menée sur une villa romaine des Alpes-Maritimes a permis, entre autres, la découverte de nombreux surcuits céramiques. L'analyse du contexte et du milieu naturel permettent de concevoir une production locale. Quatre catégories de fabrication sont définies (céramiques communes à pâte claire ou sombre ; petites amphores ; tuiles). Cet atelier devait être destiné pour l'essentiel à la confection des petites amphores répondant aux besoins économiques de la villa (ou de plusieurs ?) ; il a produit durant un bref laps de temps que l'on situera vers le milieu du Ier s. de n. è.

(Mots-clés : Céramiques, Atelier de potier, Haut-Empire romain, Mandelieu, Alpes-Maritimes)

\section{A pottery workshop of the first cent. A.D. at Mandelieu (A.-M.)}

Abstract - An excavation of urgency conducted on the site of a roman villa in Alpes-Maritimes enabled the discovery of numerous discarted pottery workshop vases. The analysis of the context and the natural environment show a local production. Four types of articles are defined : common pottery made of clear or dark clay, small amphoras, tiles. This pottery workshop must have been intented essentially for the making of small amphoras, for the economical needs of the villa (or several ?). It was in production during a brief laps of time that can be situated about the middle of the first cent. A.D.

(Key words : Potteries, Pottery workshop, Early Roman Empire, Mandelieu, Alpes-Maritimes)

1. Entre 1979 et 1981 , la fouille d'urgence menée sur le site d'une villa gallo-romaine a livré une masse importante de céramique (1). La récolte de nombreux fragments surcuits, de même que la fréquence de certaines formes de céramiques communes ou de petites amphores, attestent l'activité de potiers dans les abords immédiats du site de la villa (2).

\section{Le site (fig. 1)}

La villa est installée au sud-est de la ville actuelle de Mandelieu, dans le quartier de Minelle, au lieu-dit "NotreDame d'Avignonet" (3). Elle occupe la surface d'une petite butte qui se dégage de trois ou quatre mètres seulement audessus de la plaine alluviale et littorale, encadrée par les derniers contreforts du Massif de l'Estérel.

Deux cours d'eau limitent la partie occidentale de cette zone déprimée : l'Argentière au sud-ouest, à $400 \mathrm{~m}$ environ du site, et la Siagne à l'est, à environ $1100 \mathrm{~m}$ (4).

Le terrain naturel est un banc d'argile dont les propriétés semblent être particulièrement favorables à la production céramique (5). C'est, en même temps, un sol propice à l'agriculture (aujourd'hui, vignes et vergers) ; on ne compte pas moins de trois villae sur quatre ou cinq $\mathrm{km} 2$ (6).

Il est presque superflu d'évoquer la proximité d'une forêt pour l'approvisionnement en bois de chauffe ; les collines environnantes sont encore couvertes de résineux.

Saignée débouchant dans le Golfe de La Napoule, ouverte aux échanges avec l'arrière-pays par la vallée de la Siagne, cette basse vallée est aussi un passage obligé pour un axe littoral ouest-est ; la via per Alpes Maritimas l'emprunte très vraisemblablement (7). Parallèlement, le cabotage devait tenir une place importante dans les relations commerciales.

De toute évidence, des conditions favorables pour l'installation et le développement d'un artisanat céramique sont réunies dans cette zone. 


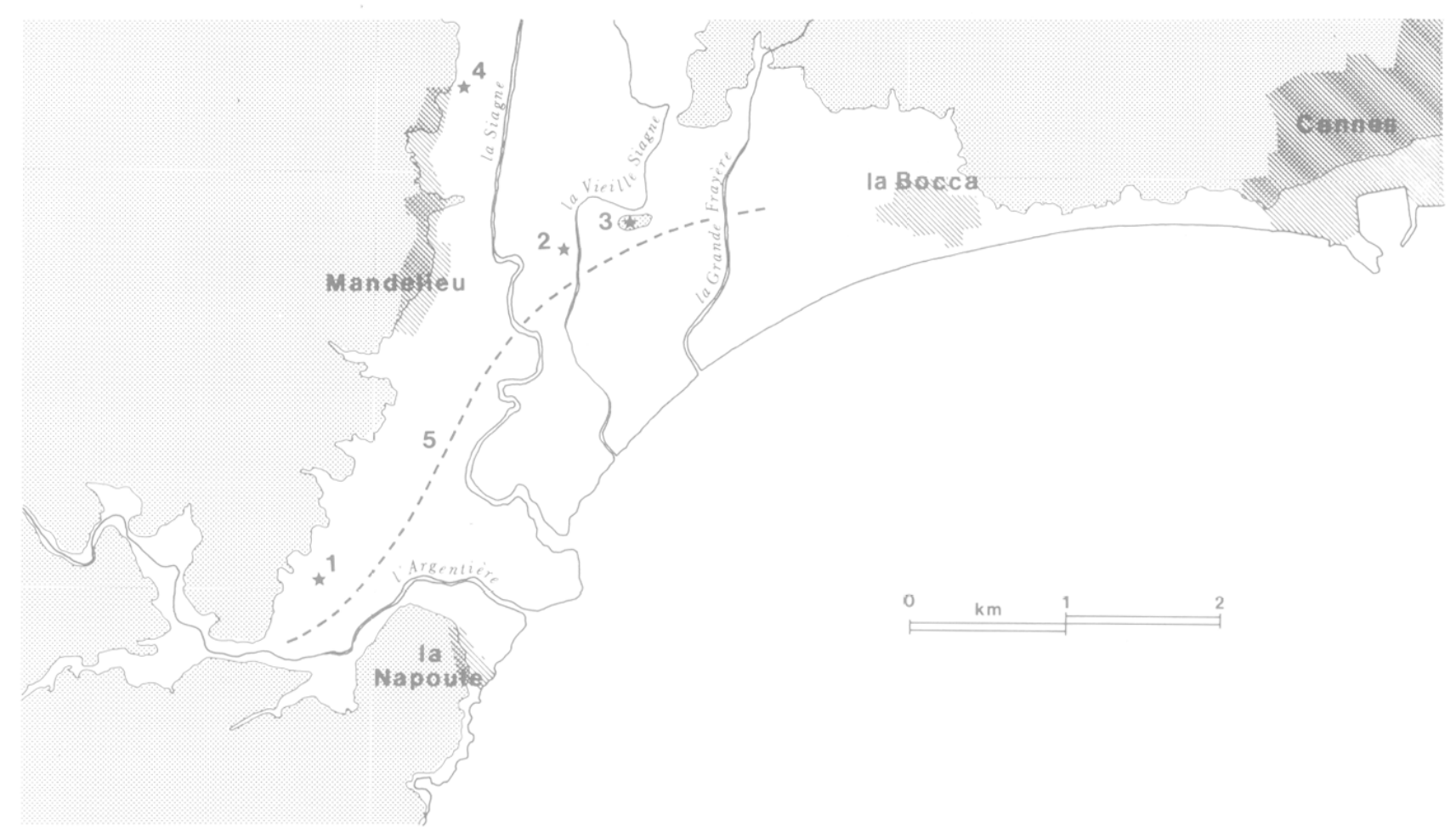

Fig. 1 - La région de Cannes - Mandelieu. 1 : villa de Mandelieu - Minelle. 2 : villa proche de la colline Saint-Cassien. 3 : prieuré de Saint-Cassien. 4 : villa du Château de la Tour. 5 : tracé possible de la voie per Alpes Maritimas (Julia Augusta). En grisé, le relief (altitude supérieure à $10 \mathrm{~m}$ ).

\section{La villa et les témoins de production céramique}

La villa, dont la recherche archéologique n'a révélé qu'une partie de la pars urbana, est édifiée au début du Ier s. de n. è. Différents remaniements transforment le plan des bâtiments et témoignent d'une occupation, sans doute ininterrompue, jusque durant le IVème s. ; à cette époque, l'un des espaces accolé aux thermes (peut-être un cellier) est aménagé en lieu de culte à mystères, sans doute un mithràeum Ce dernier est abandonné dans les dernières années du IVème s. (8).

Les objets qui nous intéressent ici, les blocs d'argile fondue, les tessons nettement surcuits, déformés, verdis (9), figurent dans des couches de remblais qui sont en grande partie remaniées pendant toute la période d'occupation de la villa. Il y a donc une difficulté pour apprécier, et la datation absolue de ce matériel, et une éventuelle évolution dans la chronologie de ces productions. En fait on ne peut guère considérer ces témoins que comme un tout et dater les débuts de la période de production en fonction de ceux d'entre eux qui apparaissent dans les couches les plus précoces.

Pour cet ensemble d'objets, la datation, certes approximative, peut correspondre au milieu du Ier s. de n. è. (10).

Le faciès des quelques formes analysables de cette production ne détonne pas, d'ailleurs, d'une période qui couvrirait les règnes de Claude à Vespasien. Ajouté à la carence chronologique, le faible nombre d'échantillons à notre disposition, en céramique commune, interdit de déce- ler une progression morphologique. En revanche, les témoins un peu plus abondants d'amphorettes prouvent, semble-t-il, une absence totale d'évolution, si ce n'est dans des détails qu'il faut plutôt chercher à expliquer par le mode de production non strictement standardisé.

Il y aura donc plutôt lieu de considérer que nous sommes en face de produits fabriqués durant une période relativement brève, limitée aux décennies qui encadrent le milieu du Ier s. de n. è.

\section{Les productions}

La quantité relativement faible des témoins de production ne nous fournit, sans aucun doute, qu'un échantillonnage bien incomplet de la panoplie des objets effectivement réalisés par l'atelier. D'autre part,' la qualité des tessons, souvent très fragmentés, ne nous donne pas toujours le moyen de restituer, ou de retrouver dans le reste des tessons recueillis, la forme commercialisée.

On se limitera donc à ne livrer que les objets dont la production est indiscutablement prouvée par des fragments surcuits. On donnera les dessins de ces ratés de cuisson (de ceux qui sont dessinables, c'est-à-dire identifiables) ; on donnera également, plus particulièrement pour les amphorettes, les équivalents qui ont été commercialisés sur place. En revanche on ne tentera pas de restituer les formes des fragments qui posent problème. 


\begin{tabular}{|c|c|c|c|c|c|c|c|c|c|}
\hline Prod. céramique & cér. comm. & cér. comm. & cćr. comm. à pâte & cér. comm. & cér. comm. à pâte & amphorettes & tegulae & imbrices & Total \\
\hline Phases & à pâte grise & à pâte brune & grise, brune ou clairc & à pâte claire & claire ou amphorettes & & & & \\
\hline $\begin{array}{l}\text { ćp. Claudienne } \\
\text { ct Néronienne }\end{array}$ & () & () & 0 & 4 & 8 & 96 & 4 & 15 & 127 \\
\hline ép. Flavienne & 1 & 0 & 0 & 10 & 3 & 10 & 1 & 0 & 25 \\
\hline IIlème et IIIème s. & 0 & 0 & 0 & 2 & 0 & 1 & 0 & 0 & 3 \\
\hline IVème s. & 0 & 21 & 1 & 42 & 5 & 25 & 2 & 4 & 100 \\
\hline postérieur & 2 & 1 & 3 & 29 & 4 & 51 & 2 & 4 & 96 \\
\hline Total & 3 & 22 & 4 & 87 & 20 & 183 & 9 & 23 & 351 \\
\hline
\end{tabular}

Fig. 2 - Tableau de répartition des fragments surcuits par catégories et par phases chronologiques.

(11):

Quatre types de produits sont nettement fabriqués

- de la céramique commune à pâte sombre ;

- de la céramique commune à pâte claire ;

- des amphorettes;

- des tuiles.

Le tableau suivant (fig. 2) donne la répartition de ces différents fragments surcuits, en fonction des grandes phases chronologiques qui résultent des données de la fouille. Il serait parfaitement irréaliste d'extrapoler, à partir de ces chiffres, sur l'aspect quantitatif des productions réellement issues de l'atelier. On retiendra essentiellement que les amphorettes, moins mal représentées, nous donnent, sans doute, des informations plus complètes que les autres séries.

\subsection{LA CERAMIQUE COMMUNE A PATE SOMBRE}

Par leur couleur, les tessons surcuits ne permettent pas systématiquement de renvoyer à des produits en pâte grise ou en pâte brune ; certains tessons même, brun foncé, appartiennent en fait à des fabrications en pâte claire ou à des amphorettes, comme le certifient les éléments de forme conservés. Nous ne prendrons donc en compte que les seuls fragments surcuits qui, essentiellement du point de vue formel, conduisent indiscutablement à des objets conçus pour être cuits en atmosphère réductrice / réductrice (12), la coloration définitive se déterminant en fonction de l'étanchéité du four obtenue durant la post-cuisson.

\subsubsection{La céramique d̀ pâte grise (fig.3)}

1 - Bord d'olla à lèvre arrondie (Esp. II C, c. 1, $n^{\circ} 1404$ ). Surcuit et déformé ; le diamètre et l'horizontale sont approximatifs.

2 - Bord d'olla à lèvre mince rabattue et aplatie vers l'extérieur ; petit épaulement marqué à la liaison col (concave) / panse (convexe); (Esp. I C, c. $7, n^{\circ} 2992$, contexte de datation : Epoque flavienne). Surcuit et déformé.

3 - Fond plat d'olla (Esp. IX C, c. 1, n² 2957). Surcuit à peine déformé.

Les deux bords renvoient à des ollae, vraisemblablement sans anse ; au Ier s. de n. è., les comparaisons ne manquent pas (13). Le fond plat pourrait tout à fait appartenir à cette même famille de vaisselle culinaire.

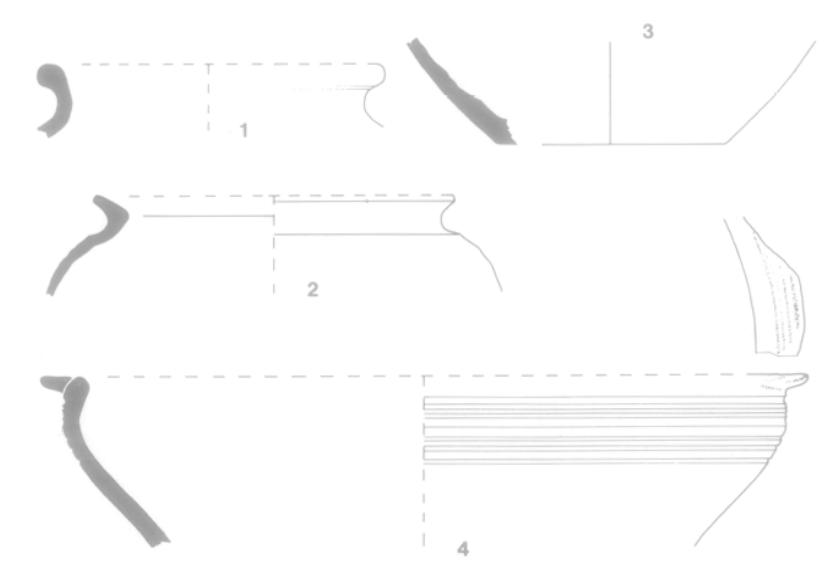

Fig. 3 - Céramique commune à pâte sombre : ratés de cuisson. 1 à 3 : pâte grise ; 4 : pâte brune (éch. 1/3).

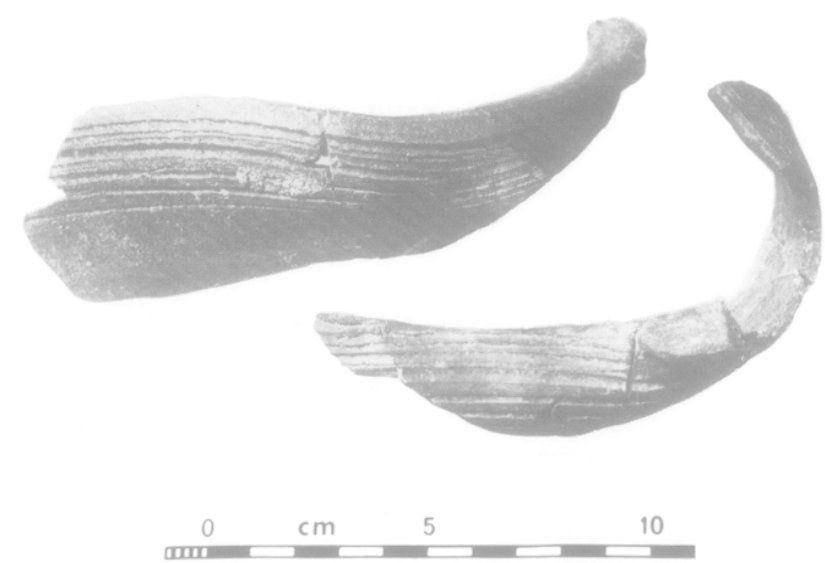

Fig. 4 - Jatte surcuite en céramique commune à pâte brune (cf. fig. $\left.3, \mathrm{n}^{\circ} 4\right)$ (cl. auteur).

\subsubsection{La céramique d pâte brune (fig. 3 et 4 )}

4- Bord de jatte munie de deux oreilles de préhension; la partie supérieure de la panse comporte, à l'extérieur, une demi-douzaine de stries ; le bord est recourbé vers l'intérieur avec une lèvre simplement arrondie (Esp. II C, c. $2, n^{\circ} 434$ ). Surcuit totalement déformé, distordu ; diamètre approximatif. Deux autres ratés de cuisson (même provenance) portent une oreille : ils représentent une seconde jatte (indessinable tant les tessons sont "fondus"). 


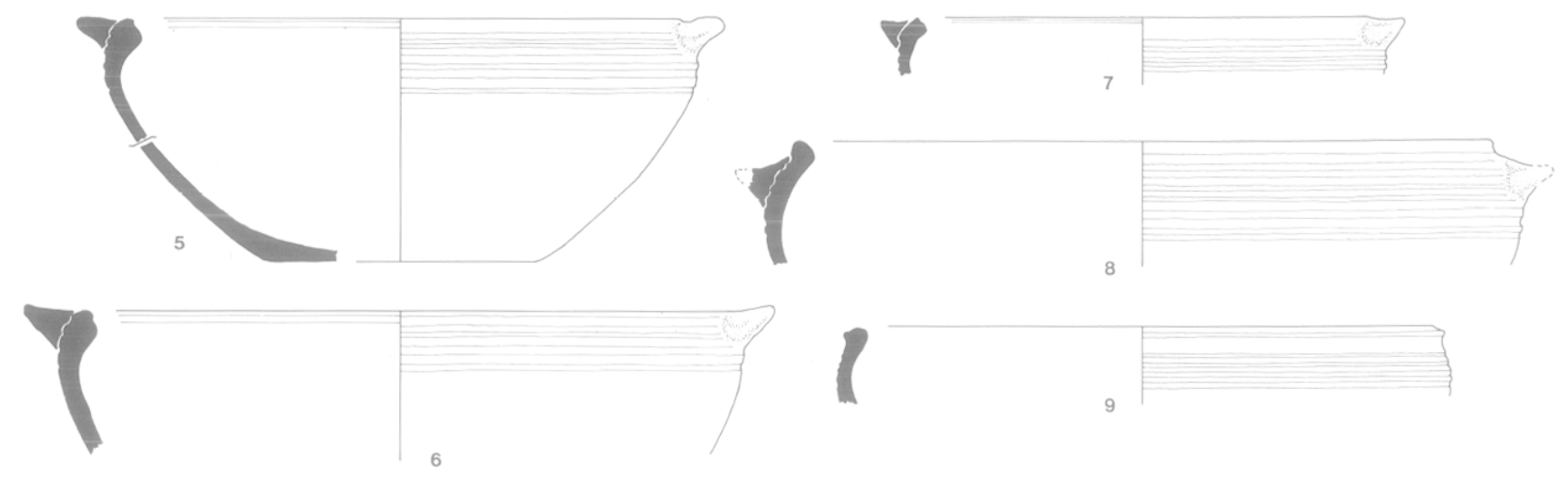

Fig. 5 - Céramique commune à pâte brune : jattes commercialisées provenant de la villa (éch. 1/3).

Ces bords appartiennent, sans conteste, à des objets réalisés en pâte brune. Après cuisson, l'argile présente les traits suivants : la pâte est grossière et inclut de minuscules grains blancs de forme arrondie, beaucoup de gros grains ivoire ou beige (jusqu'à 2 et $3 \mathrm{~mm}$ ) à arêtes vives, en tranche comme en surface, ainsi que de nombreuses paillettes à reflet argenté, surtout en surface. La couleur est brunjaune-rouge, souvent plus claire à l'intérieur (code Cailleux et Taylor : C. 48) qu'à l'extérieur (D. 43).

On dispose de plusieurs exemplaires commercialisés (fig. 5). Les trois premières jattes $\left(\mathrm{n}^{\circ} 5,6\right.$ et 7$)$ montrent un profil identique à celui du raté de cuisson, en particulier avec une lèvre simplement arrondie vers l'intérieur. L'exemplaire reconstitué graphiquement (les fragments de bord et de fond se rapportent au même vase, mais un collage de jonction n'a pu être réalisé) montre une panse galbée et légèrement tronconique, reposant sur un fond plat. Les deux autres bords $\left(n^{\circ} 8\right.$ et 9 ) se distinguent des précédents par une lèvre épaissie à l'extérieur, constituant un mince bourrelet.

La forme générale de cette jatte, de même que le "style" créé par les sillons creusés sous le bord, renvoient aux influences exercées par la tradition indigène (14).

\subsection{LA CERAMIQUE COMMUNE A PATE CLAIRE}

Le lot de tessons surcuits se rattachant à cette catégorie (15) fournit deux types de produits qui se différencient tant par des critères de qualité que par la fonction; il est donc nécessaire de distinguer les objets qui procèdent plutôt des céramiques à "parois fines" de ceux qui correspondent à des formes plus grossières et plus conformes à cette catégorie.

\subsubsection{La céramique a parois minces (fig.6)}

10 - Goulot de cruche à une anse ; le profil du col, légèrement étranglé, suggère une panse ovoïde; l'anse est peut-être plate (Esp. II G, c. 2, $\mathrm{n}^{\circ} 2551$ ). Surcuit fortement deforme ; diamètre et horizontale approximatifs.

11 - Bord de cruche ou de pot à une anse (Esp. II C, c. 5, $n^{\circ} 1439$ ). Surcuit fortement déformé.

12 - Bord de pot à large ouverture ; bord recourbé vers l'extérieur avec lèvre simplement arrondie (Esp. II C, c. 6, $n^{\circ}$ 1674). Surcuit fortement déforme.
13 - Bord de pot à large ouverture; bord recourbé vers l'extérieur avec lèvre amincie (Esp. II C, c. 6, n 1551-1552). Surcuit fortement déformé.

14 - Fragment de panse présentant une légère carène au niveau du plus fort diamètre, où s'attache la partie inférieure d'une anse, sans doute plate (Esp. II C, c. 10, $n^{\circ} 1243$; contexte de datation : Epoque flavienne). Surcuit très déformé.

15 - Fond plat muni d'un petit pied dégagé de la masse par une gorge ; bas de panse hémisphérique dont l'extérieur est lissé en facettes (Esp. II C, c. 6 et $10, n^{\circ} 1674$ et 1243 ; contexte de datation : époque flavienne). Surcuit fortement deformé.

16 - Fond plat reposant sur un petit pied sans doute dégagé par enlèvement de l'argile ; la paroi du fond parait très mince ; bas de panse hémisphérique lissé en facettes à l'extérieur (Esp. II C, c. 5 et 10, n ${ }^{\circ} 1439$ et 1242 ; contexte de datation : époque flavienne). Surcuit très peu déformé.

17 - Fond plat pourvu d'un petit pied dégagé par une gorge (Esp. II G, c. $1, \mathrm{n}^{\circ} 2603$ ). Surcuit non déformé.

Ces éléments de formes appartiennent à des petites cruches ou à des pots avec ou sans anse; ils illustrent une catégorie de vaisselle que l'on peut rencontrer aux Ier et IIème s., vaisselle qui figure sur la table pour assurer des fonctions secondaires qu'il est bien difficile de préciser. Ces objets sont tout aussi fréquents en pâte grise qu'en pâte claire : dans ce dernier cas, ils sont généralement revêtus d'un engobe non grèsé. Tous semblent appartenir à des vases fermés; ils ne se substituent donc pas aux formes les plus fréquentes de la céramique a "parois fines".

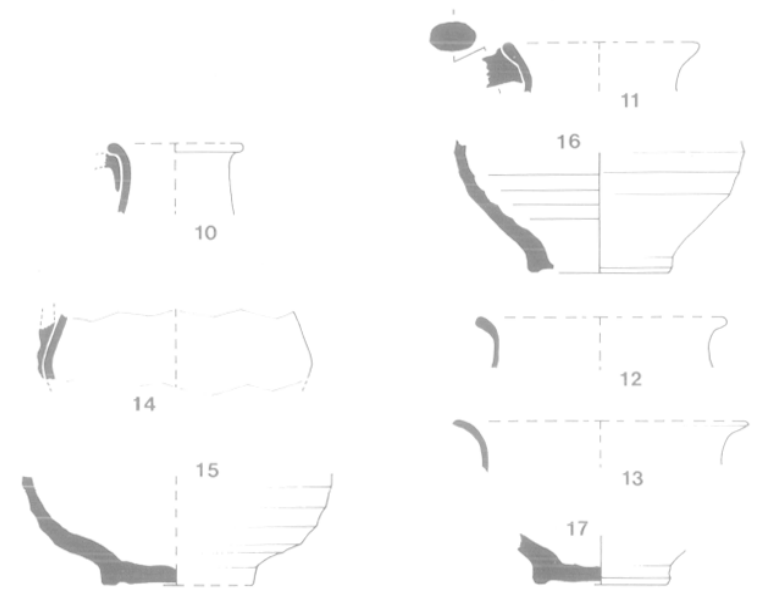

Fig. 6 - Céramique commune à pâte claire : ratés de cuisson. Vases fermés à parois minces. 


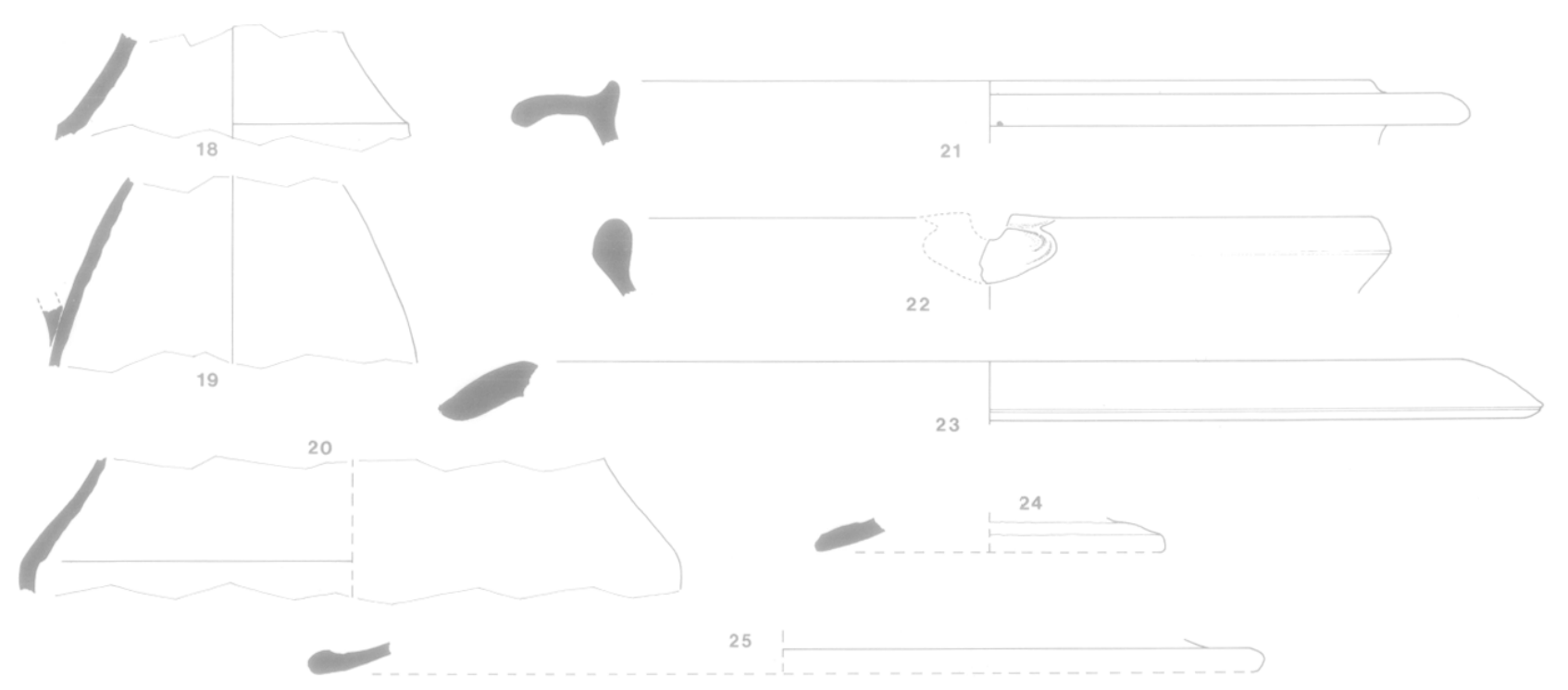

Fig. 7 - Céramique commune à pâte claire : ratés de cuisson. Cruches, mortiers et couvercles (éch. 1/3).

Faute d'objets suffisamment bien conservés, y compris dans le lot de tessons commercialisés sur le site, nous ne pouvons présenter ou proposer de formes équivalentes moins incomplètes.

\subsubsection{Cruches, mortiers et couvercles (fig.7)}

18 - Col de cruche (?) à épaulement fortement marqué ; ouverture réduite ; pas de trace d'anse (Esp. X, c. 3, n 2896 ; contexte de datation : Tibère - Claude ?). Surcuit non déformé.

19 - Haut de panse d'une cruche piriforme munie d'une anse plate (Esp. XX C, c. $\left.2, n^{\circ} 2974\right)$. Surcuit non déformé.

20. Epaule de grande cruche suggérant un large col (Esp. IX C, c. 2, $n^{\circ} 2945$ ). Surcuit déformé.

Ces trois éléments correspondent, vraisemblablement, à des cruches à une anse. La grandeur des tessons interdit de trouver, à coup sûr, des récipients identiques dans un catalogue de formes issues, sans doute, de conceptions presque exclusivement italiques (16).

21 - Bord de mortier à collerette ; le bord est mince, légèrement rentrant, bien dégagé au-dessus de la collerette ; celle-ci, horizontale près du bord, se recourbe et se termine par une lèvre simplement arrondie (Esp.IX C, c. $\left.1, \mathrm{n}^{\circ} 2977\right)$. Très cuit, non déformé.

22 - Bord de mortier (ou de coupe) à bec verseur ; la lèvre est épaissie et arrondie ; la paroi de la panse parait assez fine (Esp. I C, c. 8, $n^{\circ} 1746$; contexte de datation : époque flavienne). Très cuit, non déformé.

Le premier bord correspond à un type de mortiers très fréquent sur un grand nombre de sites gallo-romains, dès le milieu du Ier s. de n. è. ; le versoir n'est pas conservé sur ce fragment (17). Le second bord, en revanche, ne connait pas d'équivalent parfait ; pourtant il s'agit vraisemblablement d'un mortier (ou coupe) "à listel" qui se rencontre aussi en grand nombre, en particulier sur les sites du littoral nordméditerranéen (18).

23 - Large bord rabatu fortement vers l'extérieur (ou collerette ?) ; la lèvre est amincie (Esp. II G, c. 1, $n^{\circ} 2641$ ). Surcuit non déformé.

On ne peut préciser de quel type d'objet il s'agit, du bord d'une grande coupe (à panse évasée), du marli d'un grand pot (à panse cylindrique, comme on en trouve abon- damment sur le site), ou encore de la collerette d'un mortier.

24 - Couvercle de petite dimension; la lèvre est seulement arrondie dans le prolongement de la paroi (Esp. I B, c. 1, ${ }^{\circ} 1935$ ). Surcuit légèrement déformé.

25 - Couvercle de grand diamètre, à lèvre formant un bourrelet arrondi à l'extérieur (Esp. II F, c. 1, n 1976). Surcuit légèrement déformé.

C'est, là encore, un objet très fréquent ; les parois, rectilignes ou légèrement courbes, se rejoignent vers un bouton central de préhension. Conçu, ici, en pâte claire, le couvercle est le plus souvent en pâte brune.

\subsection{LES AMPHORETTES}

Comme l'indique le tableau de comptage (fig. 2), c'est la catégorie de produits la mieux représentée (près de $55 \%$ ). Nous verrons qu'elle l'est aussi dans l'ensemble des fragments provenant des conteneurs commercialisés. Observons tout d'abord la série des surcuits (fig. 8).

26 - Amphoretue à anses plates creusces d'un sillon central ; l'anse est coudé fortement. Le corps est fusele et un Epaulement arrondi marque le haut de la panse au niveau de l'attache de l'anse. Le col est cylindrique, surmonté d'un bord triangulaire décalé vers l'extérieur at formant un court bandeau creusé d'une fine gorge médiane. L'amphorette repose sur une pointe. La hauteur conservee est de $34 \mathrm{~cm}$ et permet de supposer une hauteur totale comprise entre 42 et $45 \mathrm{~cm}$; diam. de l'ouv. : $8,5 \mathrm{~cm}$; diam. max. à l'epaul. : $15,2 \mathrm{~cm} ; \mathrm{h}$ anse : $8 \mathrm{~cm}$ (Esp. I B Contrefort, $\mathrm{n}^{\circ} 432$ et $2744 ; 27$ fragments ; contexte de datation : milieu Ier s. ?). Surcuit un peu déformé mais dessinable sans erreur (fig. 9).

27 - Col d'amphorette du même type avec les mêmes caractéristiques ; sous le bord et à la hauteur de l'autache de l'anse, fin filet saillant sur le col (à $2,5 \mathrm{~cm}$ sous l'ouverture); diam. à l'ouv. : env. $9,2 \mathrm{~cm}$; diam. max. à l'epaul. : env, $15,4 \mathrm{~cm} ; \mathrm{h}$ anse : $9,5 \mathrm{~cm}$ (Esp. I B Contrefort, $\mathrm{n}^{\circ} 428$, 1909 et $2744 ; 7$ fragments ; contexte de datation : milieu Ier s. ?). Surcuit très déformé.

28 - Pointe pleine appartenant très certainement au type préçdent (Esp. DX A, c. 1 a, n 2998). Surcuit non déformé.

29 - Bord légèrement évasé, à double próminence formant bandeau. La lèvre, aplatie, est tiré à l'oblique vers l'extérieur, déterminant un méplat incliné vers l'intérieur. Un bourrelet d'argile forme un cordon sur le col à 


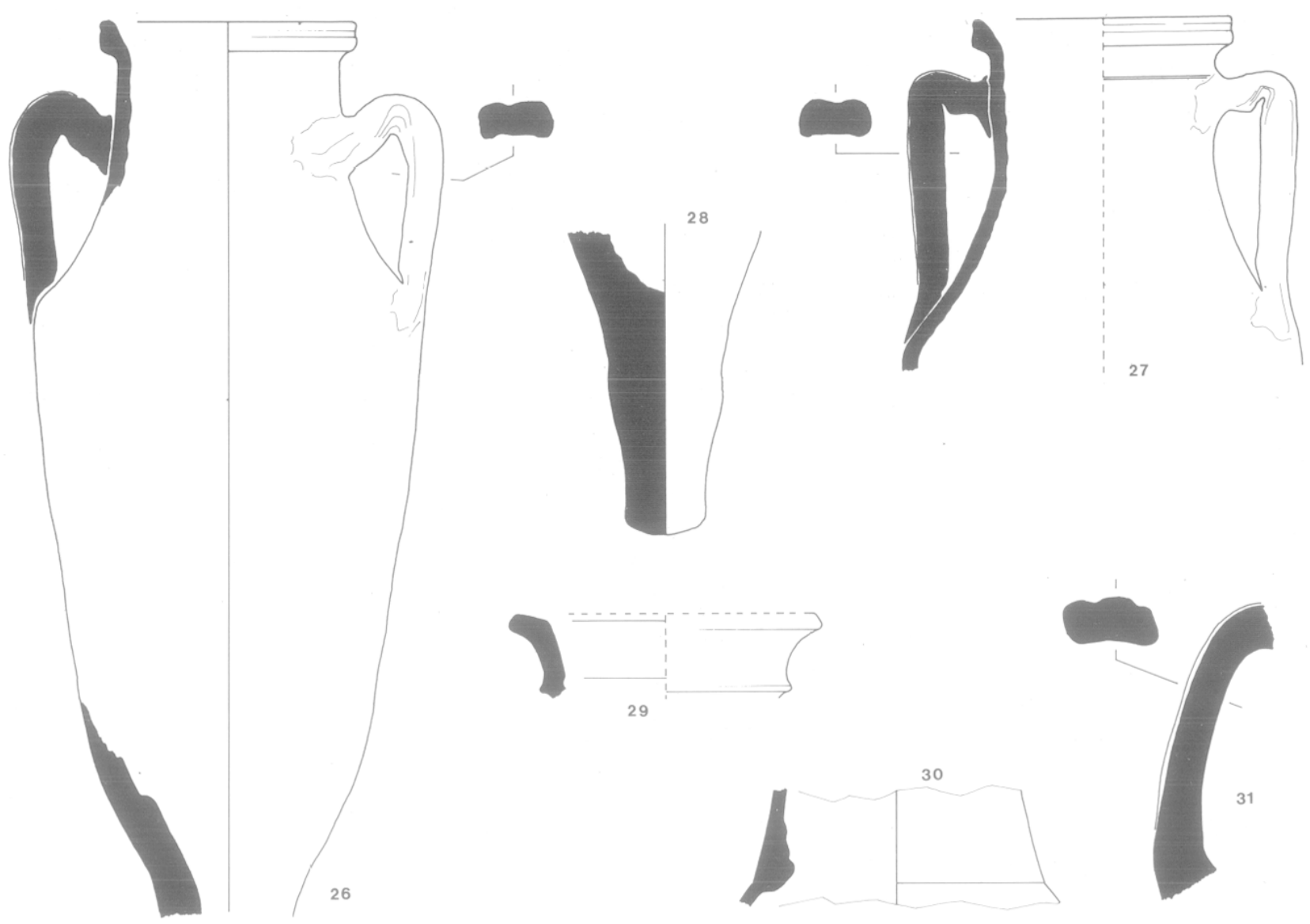

Fig. 8 - Amphoreutes : ratés de cuisson. 26 à 28 : type I; 29 à 31 : type II (éch. 1/3).

$3 \mathrm{~cm}$ sous l'ouverture ; diam. à l'ouv. : env. $9 \mathrm{~cm}$ (Esp. I B Contrefort, $n^{\circ} 1828$ et 2744 ; contexte de datation : milieu ler s. ?). Surcuit déformé (le tesson donne un diamètre légèrement inférieur mais il est replié par la surcuisson).

30 - Fragment assurant la liaison entre le col et la panse ; le changement d'orientation de la paroi est marque, à l'extérieur, par un sillon ; à l'intérieur, bourrelet forme par le racoord à l'argile (Esp. II A, $\mathrm{n}^{\circ}$ 6011, hors stratigraphie). Surcuit non déformé.

31 - Anse plate creusée de deux sillons peu profonds (Esp. II A, c. 1, $n^{\circ}$ 2923). Surcuit peu déformé.

Ces ratés de cuisson permettent de déterminer deux séries d'amphorettes. Pour cette étude, nous nous appuierons sur l'examen des amphorettes non surcuites retrouvées sur le site. Elles présentent toutes, après cuisson, une pâte qui répond à la description suivante : argile très fine contenant une grande quantité de minuscules grains blancs de forme arrondie (peu visibles à l'oeil), des grains beiges ou marrons, à arêtes franches (visibles) et quelques paillettes à reflet argenté (exceptionnellement à reflet doré); la couleur répartit les tessons en deux lots bien distincts : 1'un uniformément rouge clair (code Cailleux et Taylor: C. 38), l'autre rouge clair et jaune très pâle (B. 61) ; pour les premiers, la pâte est très tendre, très pulvérulente ; pour les autres elle est mieux cuite et plus dure.

\subsubsection{L'amphorette du type I}

(fig. $8, n^{\circ} 26,27,28$; fig. 9 et 10 )

Revenons, pour les préciser, sur les critères d'identifi- cation de la forme. Le corps (à parois épaisses, $1 \mathrm{~cm}$ env. ) et le col sont façonnés en une seule fois comme l'indique l'absence de collage au-dessus de l'épaulement ; une seule exception à cette règle est illustrée par le col n' 37 qui semble être un cas unique sur le site. La pointe est pleine, l'extrémité étant arrondie. L'épaulement, arrondi aussi, est peu marqué ; pourtant, sur presque tous les exemplaires, on constate que le traitement de surface n'est pas le même pour la panse et pour le col. En règle générale, ce dernier est lissé plus soigneusement, ce qui marque à l'oeil une ligne d'épaulement ; celle-ci est, d'ailleurs, parfois clairement indiquée par un coup d'estèque qui crée un léger angle à la surface. On peut même trouver deux fines stries $\left(n^{\circ} 35\right)$ ou encore une petite gorge $\left(n^{\circ} 37\right)$. En revanche, sous le bord, le filet saillant est toujours présent ; c'est à ce niveau que se greffe la partie supérieure de l'anse. Sur le méplat vertical du bord en bandeau, la faible gorge ne se retrouve pas systématiquement et ne semble pas être un détail volontairement réalisé ou obtenu. Les anses sont toujours plates et creusées d'un sillon central plus ou moins profond, tracé au doigt ; le coude de l'anse peut présenter des fractures dans la pâte tant le ruban d'argile est replié sur lui-même.

Dimensions mesurées (en $\mathrm{mm}$ ) sur une dizaine d'exemplaires : - hauteur totale (19) : 583

- diamètre maximum : entre 150 et 180 (moy. : 159,75; ecart-type : 13,7) - diamètre extérieur à l'ouverture : entre 100 e 123 (moy. : 108 ; écarttype : 7)

- hauteur du bandeau de la lèvre : entre 12 et 16 (moy. : 13,7; écart-type : 1,4) 
- hauteur de l'attache supérieure de l'anse sous la lèvre : entre 19 et 28 (moy. : 23 ; écart-type : 3 )

- hauteur de l'anse : entre 85 et 126 (moy. : 104,8; écart-type : 14,9)

Cette forme est représentée sur le site par plusieurs centaines de fragments (20) mais les cols entiers se comptent presque sur les doigts des deux mains ; ceci limite considérablement le commentaire. Y a-t-il un seul module ? Les écarts de dimensions sont-ils le résultat d'une production qui, tout en étant relativement standardisée, manque un peu de rigueur, ou bien pourrait-on déceler deux ou trois constantes correspondant à autant de modules ? On ne le peut et on doit se contenter de constater ces fluctuations qui touchent la plupart des paramètres et, plus particulièrement, la hauteur des anses, domaine où, justement, la copie conforme dépend en bonne partie de la rigueur du potier (21).

Cette amphorette a une très faible capacité : 3,28 I (22) ou, peut-être, un peu moins (23).

Conteneur de forme pourtant bien typé et facilement identifiable, il ne semble pas qu'on le rencontre sur les sites régionaux : il est absent à Fréjus comme à Vintimille (24). On ne le retrouve pas, non plus, sur des sites plus éloignés.

\subsubsection{L'amphorette du type II (fig.8, $\mathrm{n}^{\circ} 29,30$ et 31 )}

La production de cette petite amphore est attestée par un faible nombre de fragments surcuits, et seulement par un bord ( $\left.n^{\circ} 29\right)$. Si l'on ne disposait que de ces éléments, un bord, un fragment assurant la liaison col / panse $\left(n^{\circ} 30\right)$ et une anse $\left(n^{\circ} 31\right)$, on ne serait guère en mesure d'envisager cette série. Mais le bord surcuit et déformé justifie, à lui seul, que l'on soit attentif aux formes équivalentes commercialisées sur le site, et même qu'on les utilise pour définir la forme.

Comme pour l'amphorette du type I, les tessons de cette petite amphore se comptent aussi par centaines (25), sans qu'on ait forcément la chance, là encore, de disposer de nombreux fragments étudiables. Ces tessons font apparaitre deux variantes bien distinctes.

Il s'agit d'amphorettes à "fond plat", c'est-à-dire reposant sur un fond annulaire, dont les points communs sont le corps ovoïdc, le col haut surmonté d'un bord en bandeau, et les anses plates. Les differences se situent dans le profil de la liaison col / panse et dans celui du col lui-même, dans les détails du bord et, peut-être, dans les anses.

N'ayant en main qu'un seul bord surcuit, ce sont ces très nombreux fragments de petites amphores, de l'une et l'autre variantes, qui nous incitent à considérer qu'elles sont, sans le moindre doute, produites sur place.

\section{LE TYPE II A (fig. 11)}

C'est, apparemment, l'amphorette la moins bien représentée des deux variantes.

Le col, cylindrique, ou presque cylindrique, forme un angle bien marqué à son point d'attache avec la panse ; c'est un col tourné à part et rajouté comme l'indique, au niveau de cette jonction, le bourrelet interne d'argile. A l'extérieur, ce raccord est systématiquement souligné par un sillon creusé dans l'argile. Le bord forme un bandeau,

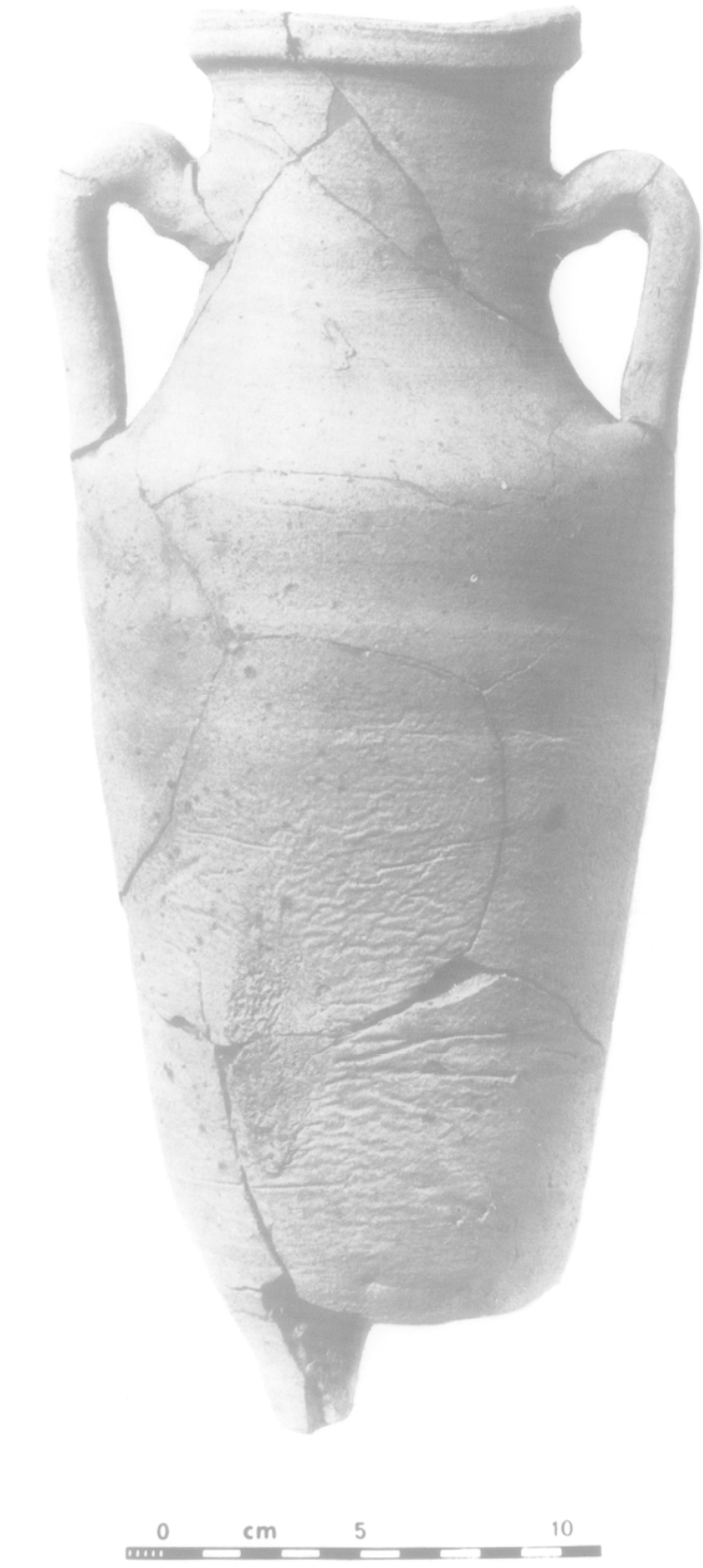

Fig. 9 - Amphorette surcuite du type I ( $c f$. fig. $8, \mathrm{n}^{\circ} 26$ ) (cl. auteur).

résultat de deux inflexions imposées à la pâte. L'inflexion inférieure détermine, à l'extérieur, un cordon, et à l'intérieur une gorge. L'inflexion supérieure a été accompagnée d'un aplatissement de la lèvre qui saille largement vers l'extérieur ; ce méplat de la face supérieure est généralement incliné vers l'intérieur. La réalisation de ce bord en forme de manchon conserve toujours à la paroi interne un plan vertical ou plus ou moins vertical. Les anses, plates et larges (entre 38 et $45 \mathrm{~mm}$ ) sont, en principe, creusées de deux ou trois sillons peu profonds tracés au doigt (l'exemplaire $n^{\circ} 42$ est donc, peut-être, un intrus). Elles s'attachent immédiatement sous le cordon du col. Le diamètre maximum se place dans la partie supérieure de la panse. Le fond, en l'état actuel du matériel, ne fournit pas de critère distinctif de celui du type II B. 


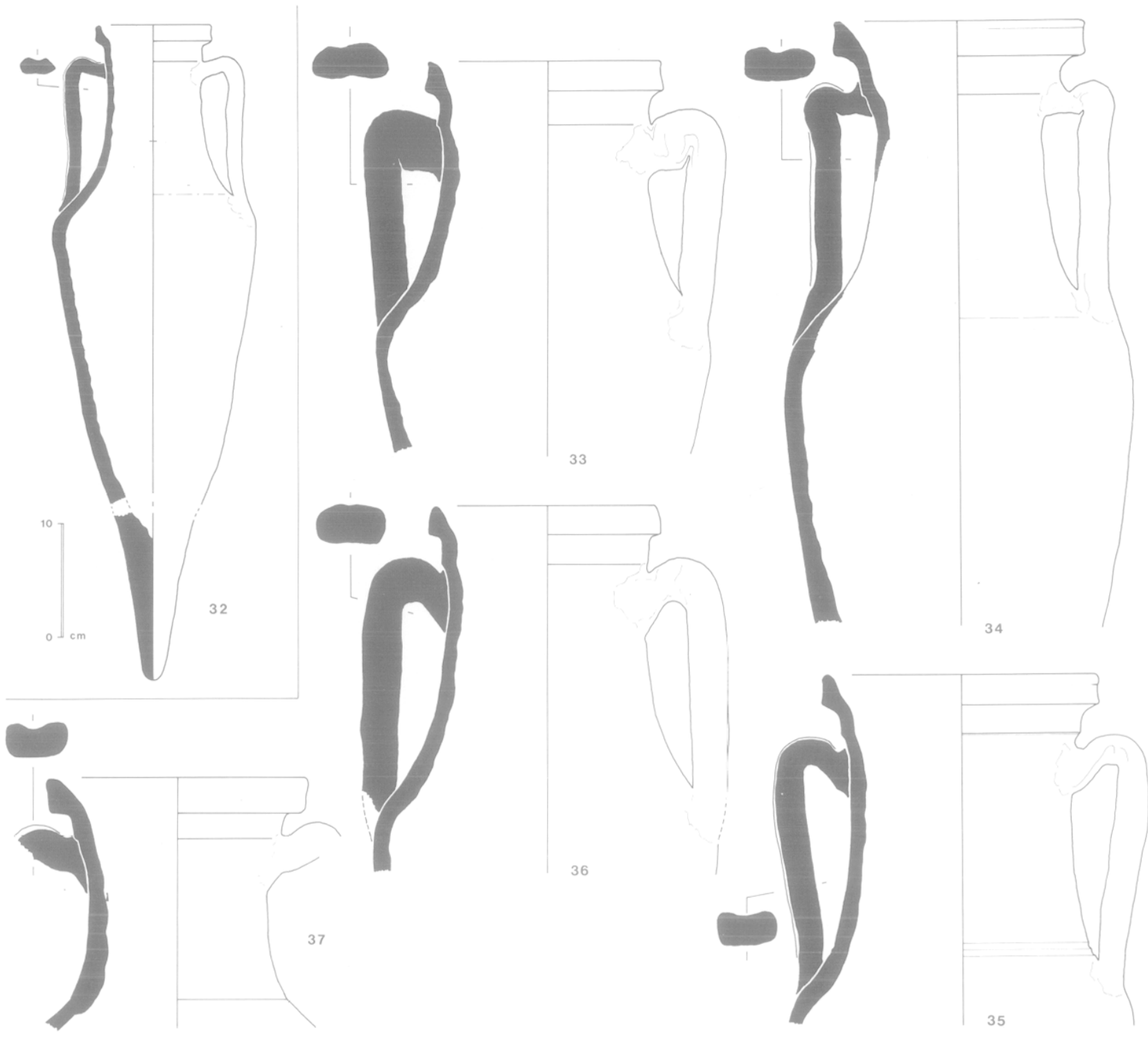

Fig. 10 - Amphorettes du type I, commercialisées et provenant de la villa (éch. 1/3, sauf $n^{\circ} 32$ ). res :

Dimensions mesurées (en mm) sur une demi-douzaine d'exemplai-

- hauteur totale (26) : 474

- diamètre maximum (26) : 287

- diamètre extérieur à l'ouverture : entre 122 et 145 (moy. : 131,4; écarttype : 14,5 )

- largeur du méplat de la lèvre : entre 9 et 16 (moy. : 11,8 ; écart-type : 3,4)

- hauteur du cordon à la lèvre : entre 25 et 28 (moy. : 26,4 ; écart-type : 1,2)

- hauteur de l'attache supérieure de l'anse sous la lèvre : entre 24 et 31 (moy. : 28 ; Écart-type : 3,5 )

- diamètre du fond : entre 110 et 130

On manque d'exemplaires suffisamment bien conservés pour affirmer que la morphologie de ces petites ampores est bien cadrée dans ces mesures ; la comparaison des dessins de cols (fig. $11, n^{\circ} 39$ et 40 par ex.) montre les nuances à imaginer dans l'ampleur de ces conteneurs et donc, vraisemblablement, dans leur volume. Les mesures réalisées pour l'exemplaire $\mathrm{n}^{\circ} 38$ donnent une capacité de 12,921 (27).
La silhouette de cette petite amphore rappelle certaines productions de l'Hérault, à Aspiran (28), et des Bouches-du-Rhône, à Velaux (29); on classe ces produits dans la forme Gauloise 7 (30). Ces deux ateliers ont justement réalisé des amphores de petit module avec des dimensions assez identiques à celles de Mandelieu - Minelle. Mais, pas plus que pour le type I, on ne trouve de comparaisons parfaites pour ce type II A.

\section{LE TYPE II B (fig. 12)}

Si la panse de cette amphorette présente une forme ovoïde, avec une moitié inférieure parfaitement comparable à celle du type II A, la moitié supérieure s'en distingue par un galbe moins prononcé dû à une liaison col / panse de nature differrente ; celle-ci se fait sans transition, en suivant la courbure de la panse qui s'inverse, passant d'un profil convexe à un profil concave qui prolonge le col, haut, en forme de diabolo. Ce dernier est tourné à part et soudé au niveau d'un bourrelet interne d'argile qui subsiste au terme de cette opération. 

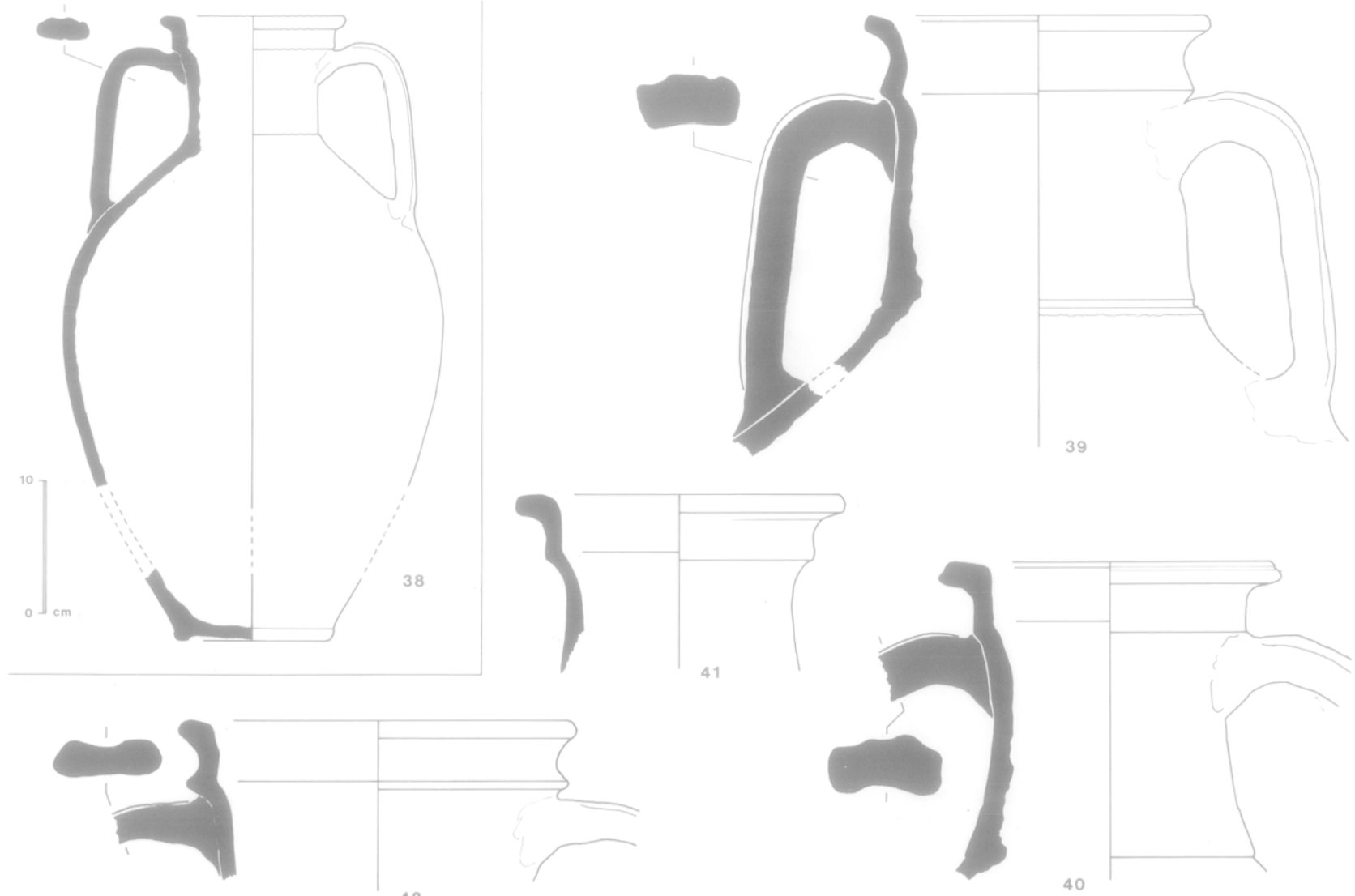

Fig. 11 - Amphorettes du type II $A$, commercialisées et provenant de la villa (éch. 1/3, sauf $n^{\circ} 38$ ).

Le diamètre maximum se situe à mi-hauteur. Le bord, comme pour le type II A, est constitué d'un bandeau résultant de deux inflexions produites dans la pâte, l'une déterminant un cordon sur le col et l'autre une lèvre aplatie et étirée à l'extérieur. C'est donc un bord comparable, sinon identique, qui encercle les ouvertures des deux variantes. Pourtant, deux différences semblent se dégager, même si elles ne sont pas systématiques. D'une part, des moulurations secondaires peuvent $s$ 'intercaler entre les deux proéminences du bandeau : ce sont des gorges ou des petits bourrelets qui ne se suivent pas régulièrement sur toute la circonférence ; d'autre part, la paroi intérieure du bandeau, en baïonnette, n'est pas toujours verticale : cette paroi peut être évasée, dans le prolongement oblique de celle du col.

Un autre trait distinctif se remarque au point d'attache supérieur de l'anse qui ne se trouve pas toujours immédiatement sous le cordon du col, mais un peu plus bas. L'anse, quant à elle, peut être creusée de deux ou trois sillons réalisés au doigt ; elle présente parfois quatre sillons creusés, dans ce cas, avec un bâtonnet.

Sur au moins un exemplaire, la panse est largement scarifiée, à cru, au niveau de l'attache inférieure des anses, pour renforcer la soudure.

Dimensions mesurées (en $\mathrm{mm}$ ) sur huit exemplaires :

- hauteur totale (31) : 505

- diamètre maximum (31) : 276

- diamètre extérieur à l'ouverture : entre 106 et 151 (moy. : 121,3 ; écarttype : 14,9)

- largeur du méplat de la lèvre : entre 10 et 18 (moy. : 14,4 ; écart-type : $2,7)$
- hauteur du cordon à la lèvre : entre 20 et 30 (moy. : 26,3 ; écart-type : 4) - hauteur de l'attache supérieure de l'anse sous la lèvre : entre 24 et 40 (moy. : 33,8 ; écart-type : 5,2 )

- diamètre du fond : entre 110 et 130

C'est donc une petite amphore un peu plus haute et un peu moins large que la précédente. Sa capacité, calculée sur l'exemplaire reconstitué graphiquement $\left(n^{\circ} 43\right)$, est de $13,121(32)$.

On peut trouver quelques similitudes avec une des petites amphores produites à Velaux (33), bien que pour cette dernière la liaison col / panse soit plus marquée, le haut de panse plus trapu, requérant une anse plus courte. La comparaison est tout aussi peu satisfaisante avec une des amphorettes produites à Fréjus (34).

\section{AUTRES AMPHORETTES (fig. 13)}

Dans le reste des tessons surcuits on trouve encore trois lèvres d'amphorettes.

50 - Partie supérieure d'un col cylindrique surmonté d'une lèvre en bourrelet arrondi vers l'extérieur ; anse plate creusée de trois sillons peu profonds (Esp. VIII A, c. $2, n^{\circ} 2863$ ). Surcuit déformé ; le diamètre et l'horizontale sont approximatifs.

51 - Partie supérieure d'un col en forme de diabolo, surmonté d'une lèvre aplatie ; anse plate creusee d'un sillon central bien marque (Esp. I C, c. 3, $\left.n^{\circ} 1866\right)$. Surcuit non déformé.

52 - Lèvre en pointe étirée vers l'extérieur (Esp. II C, c. 6, $n^{\circ} 1510$ ). Surcuit non deformé. 


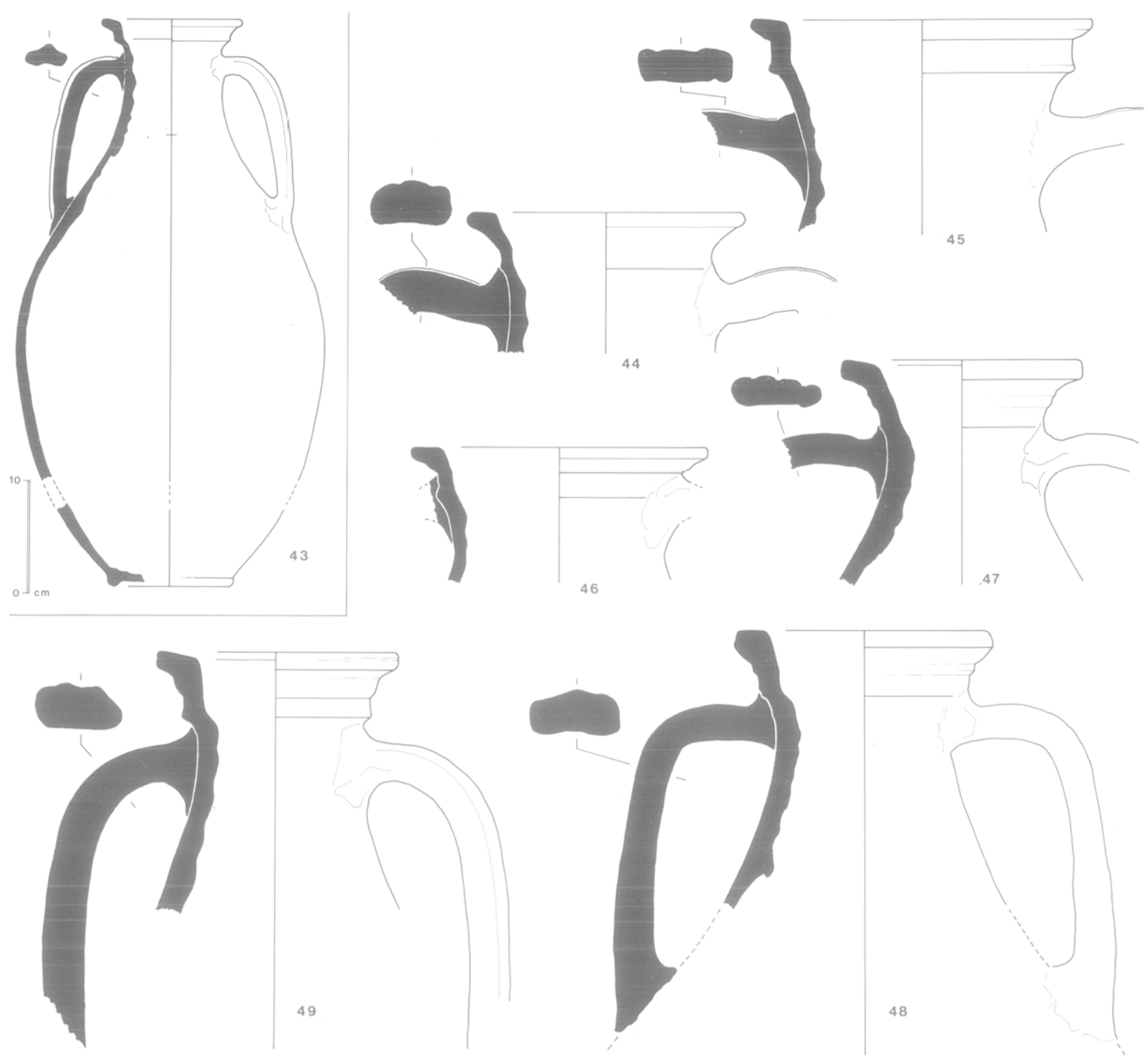

Fig. 12 - Amphorettes du type II $B$, commercialisées et provenant de la villa (éch. 1/3, sauf $\mathrm{n}^{\circ} 43$ ).

Ces lèvres témoignent de la production de petites amphores de formes différentes et variées ; pourtant, un rapport existe avec celles des types II A et II $\mathrm{B}:$ le $\mathrm{n}^{\circ} 50$ présente une anse de section identique.

\section{4.... ET LES TUILES}

Ont également été récupérés plusieurs fragments surcuits de tuiles, aussi bien des tegulae que des imbrices. Ces surcuits sont-ils la preuve que l'atelier produisait des tuiles pour les besoins locaux ? Nous ne pouvons l'affirmer et nous émettons même un doute, car la tuile, et en particulier la tuile plate (qui n'est cependant pas la plus abondante dans notre lot), est un matériau qui peut faire partie de l'équipement d'un four. Elle peut subir la surcuisson et les déformations occasionnées par une surchauffe au même ti- tre qu'un chargement de céramique. La fabrication de ces tuiles est donc, seulement, très vraisemhlable.

\section{Production céramique et économie locale}

Il ne peut y avoir le moindre doute sur l'implantation d'un atelier à proximité de la villa ; en tout cas il n'est pas inclus dans les bâtiments mis au jour (mais seule une partie de l'habitat - env. $1200 \mathrm{~m} 2$ - a été explorée). En toute logique ce serait en direction du cours de l'Argentière qu'il faudrait chercher à localiser cet atelier où ont pu s'approvi- 


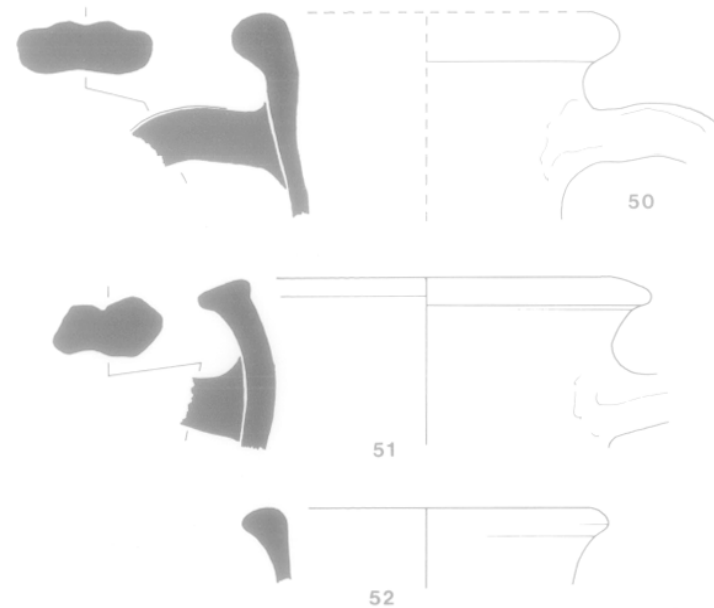

Fig. 13 - Lèvres d'amphorettes de types divers : ratés de cuisson (éch.1/3)

sionner en remblais ceux qui entreprirent, vers le milieu du Ier $\mathrm{s}$. de n. è., de remanier l'habitat; ils ne s'en privèrent d'ailleurs pas puisque l'on retrouve ces terres chargées de surcuits sur plus de la moitié de la surface du site (35).

\subsection{UN ATELIER}

A côté des tessons surcuits dont une bonne partie est recensée ci-dessus, on remarque aussi un grand nombre de déchets ou d'éléments divers se rapportant à un atelier.

Ce sont, tout d'abord (fig. 14) :
- des blocs de pierre recouverts de coulures d'argile qui, plus que les tuiles déjà mentionnées, appartiennent très certainement à l'architecture d'un four ;

- une plaque d'argile, rougie, percée d'un trou, qui pcut correspondre à une portion de sole ;

- des blocs d'argile dans lesquels des traces de doigts indiquent clairement qu'ils ont été pétris et malaxés ; surcuits d'un côté, mécuits de l'autre, ils représentent sûrement le lien argileux d'étanchéité appliqué sur les parois du four.

(fig. 15):

Ce sont, ensuite, des objets liés au façonnage des vases

53 - Disque en terre cuite (diam. : $14,6 \mathrm{~cm}$ ), percé d'un trou central (diam. : $3 \mathrm{~cm})$; incomplet et partiellement éclate $($ ep. : $3 \mathrm{~cm}$ ) (Esp. IX A, c. $2 \mathrm{~b}, \mathrm{n}^{\circ} 3005$; contexte de datation : époque flavienne).

54 - Disque en terre cuite (diam. : $11 \mathrm{~cm}$ ), percé d' un trou central (diam. : $3,2 \mathrm{~cm}$ ); incomplet (Gp. : $2,4 \mathrm{~cm}$ ) (Esp. VIII A, c.4, $\mathrm{n}^{\circ} 1152$; contexte de datation : milieu du ler s.).

55 - Cylindre creux de forme allongée ; diam. : $3,5 / 4,5 \mathrm{~cm} ; \mathrm{L}: 6,7 \mathrm{~cm}$ (Esp. DX C, c. 2, n 2950).

56 - Cylindre creux graphiquement complet ; diam. : $14,5 / 15 \mathrm{~cm} ; \mathrm{h}$ : $15 \mathrm{~cm}$ (Esp.IX C, c. 2, n² 2976).

57 . Cylindre incomplet ; diam. : $15 / 16 \mathrm{~cm}$; h conservée : $11 \mathrm{~cm}$ (Esp. II G, c. $1, n^{\circ} 2642$ ).

58 - Cylindre incomplet ; diam. : $13,5 / 14 \mathrm{~cm} ; \mathrm{h}$ conservee $: 7,8 \mathrm{~cm}$ (Esp. IX C, c. 2, $\mathrm{n}^{\circ} 2980$ ).

59 - Cylindre creux graphiquement complet ; diam. : $12 / 14,5 \mathrm{~cm} ; \mathrm{h}$ : $10,4 \mathrm{~cm}$ (Esp. IX C, c. 1, n²2954).

Les disques de terre cuite, que l'on nomme anneauxsupports, isolateurs, séparateurs, etc., sont assez bien connus. On les récupère fréquemment sur les sites d'ateliers (36) ; leur usage précis reste cependant à déterminer. A quel stade étaient-ils utilisés, entre la phase de tournage et la phase d'enfournement?
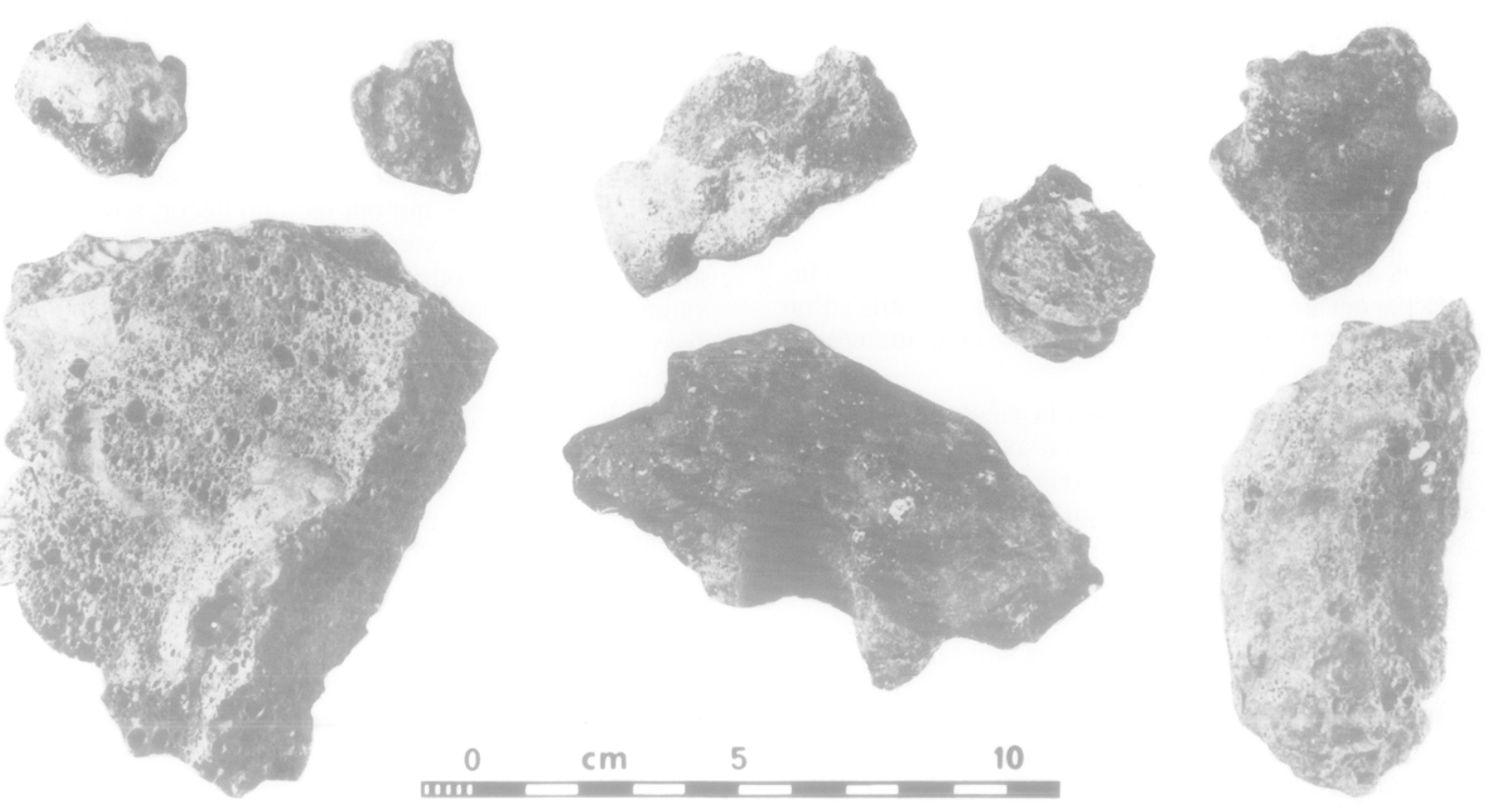

Fig. 14 - Blocs d'argile et déchets divers; au premier plan, elément de sole (?) (cl. auteur). 


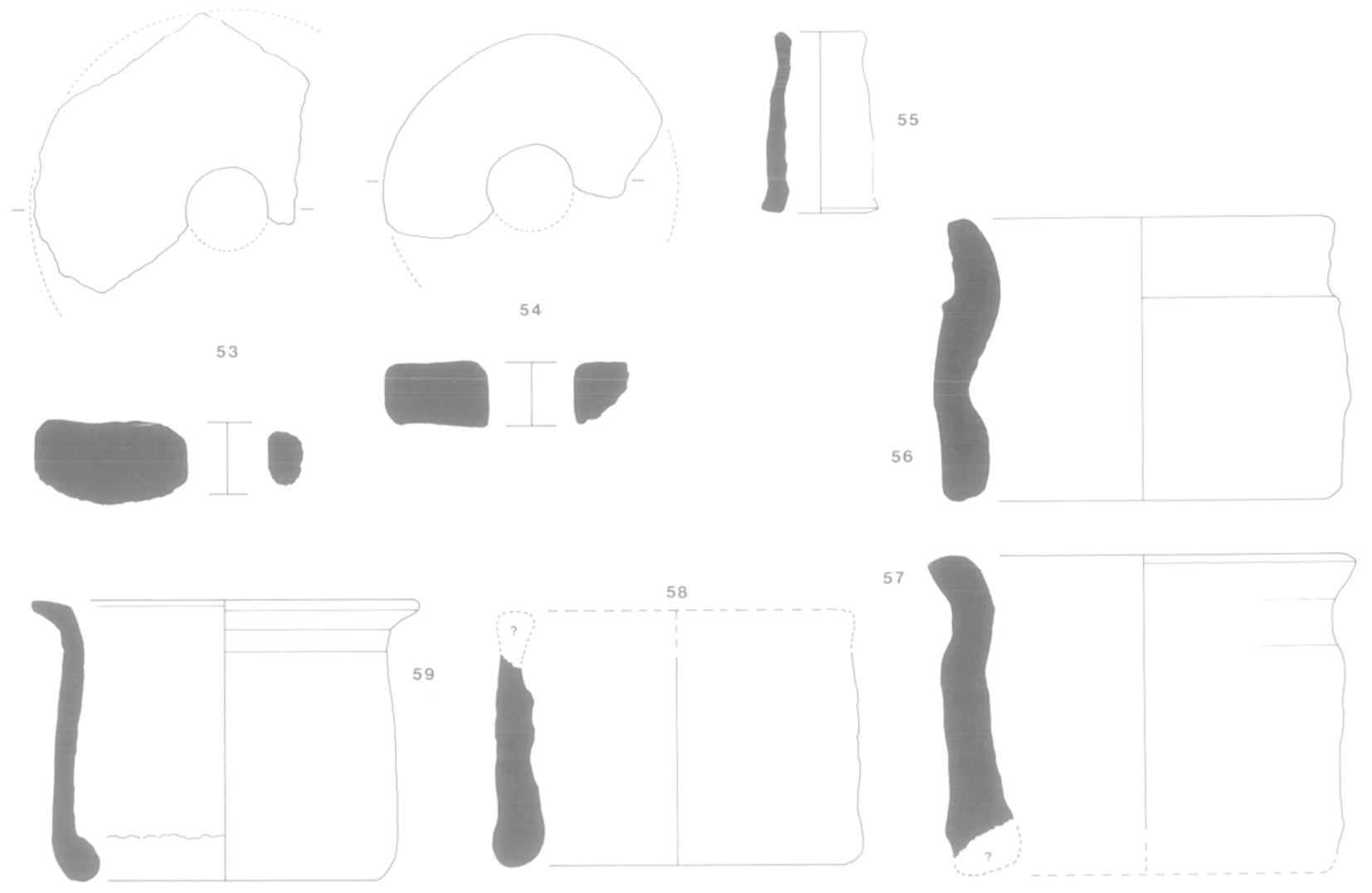

Fig. 15 - Objets liés à l'activité des potiers : disques et colliers en terre cuite provenant de la villa (éch. 1/3).

D'autres objets paraissent plus originaux ; ce sont de larges colliers en terre cuite, non surcuits eux aussi, dont la fonction reste mystérieuse. Nous n'en connaissons qu'un équivalent, à $\mathrm{La}$ Graufesenque (37). Jouent-ils un rôle assez semblable à celui des disques précédents, c'est-à-dire servent-ils, de même, à supporter des vases (38) ?

\subsection{DES PRODUITS “EN SERIE" ET DES PRODUITS "FANTAISIE"}

Nous avons donné ci-dessus la panoplie des vases fabriqués par cet atelier, en partant des tessons surcuits : d'un côté de la vaisselle commune (ou usuelle, ou courante), de l'autre des petites amphores.

On a pu constater à quel point la vaisselle commune était faiblement représentée, et nous n'avons pas dissimulé les difficultés rencontrées pour retrouver, dans le reste de la céramique exhumée, les vases équivalents; dans certains cas nous n'avons même, semble-t-il, rien retrouvé : c'est vrai pour les pots à parois minces ainsi que pour les cruches. Et cela est étonnant.

D'autant plus que pour les petites amphores le matériel est très abondant : beaucoup de surcuits, certes, mais surtout énormément d'exemplaires en usage. Il y a là véritablement une masse de tessons qui nous inciterait même à considérer que l'atelier a essentiellement produit des petites amphores, que c'était sa "spécialité". Peut-être est-ce là le fait du hasard (d'un "hasard" véhiculé en grande quantité dans les remblais).
A côté de ces témoins tangibles de production, il y a une série d'objets, en usage dans la villa, que nous devons évoquer. Ce sont des vases qui, à des titres divers (la taille, le volume, la fragilité, la qualité médiocre, l'originalité des formes ou des décors, l'extravagance, etc.) n'entrent pas dans les produits que l'on imagine, et que l'on récupère, sur d'autres sites, couramment commercialisés ; on a du mal à les considérer matériellement transportables et négociables, en tout cas à une certaine distance. Sans entrer dans une description détaillée de ces récipients, on peut dire que ce sont des coupes en pâte claire, principalement, à pâte très tendre, mal cuite, qui ont reçu un décor, soit à la roulette pour donner une sorte de guillochis très lâche, et peu soigné, soit à la barbotine travaillée au doigt. D'autres sont, toujours en pâte claire, des vases sommairement modelés, dissymétriques et, généralement, mal cuits ; un autre encore est ce vase de grande taille (fig. 16), qui concrétise si bien la fantaisie du potier (39) par son décor abusif et ses anses tarabiscotées. L'abondance de ces produits variés et uniques ne peut que renforcer, à la fois, l'hypothèse de la proximité d'un atelier, de même que l'existence de liens et d'échanges entre celui-ci et la villa.

\subsection{SURTOUT DES AMPHORETTES (fig. 17)}

Les calculs de capacité pour les amphorettes donnent les nombres suivants : 3,28 1 pour le type I, 12,92 I pour la variante $A$ du type II, et 13,121 pour la variante $B$.

Ces nombres, obtenus à partir d'un seul exemplaire de 


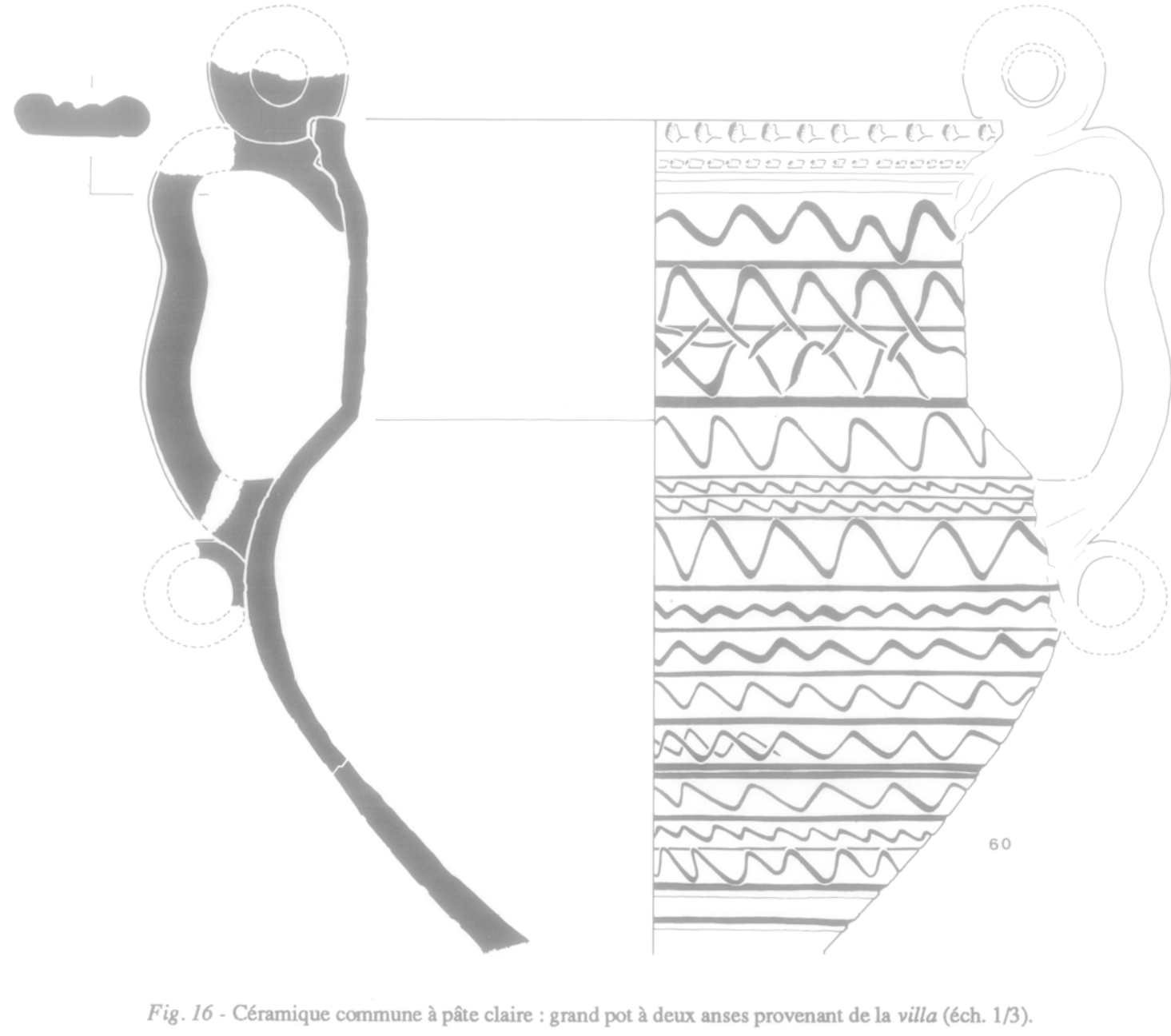

chaque type (non intact), présentent manifestement une faiblesse. Nous avons constaté, en particulier d'un col à l'autre, des différences sensibles de tailles qui peuvent éventuellement (mais non nécessairement ?) jouer sur le volume total ; des moyennes, certes, auraient sans doute permis de mieux appréhender les volumes standards. Pourtant, nous ne pouvons les évacuer purement et simplement : ils donnent, à notre avis, l'idée approximative de la contenance.

Or, il se trouve que, comparé à l'amphore canonique (ou quadrantal) de 26,261 , le type I correspond au huitième (soit 3,28 1 pour 3,28 1) et les types II à la moitié $(12,92$ l et 13,121 pour 13,131$)$. Les réserves de prudence ayant été faites, il faut bien convenir qu'il y a correspondance sinon coïncidence. Manifestement, ces amphorettes sont conçues pour entrer dans un système normalisé. Si on accepte cette constatation, elle renforce le caractère standardisé de cette production et elle permet de dire que ces amphorettes sont bien prévues pour jouer un rôle sur le marché, ce dont il était possible de douter (du fait de leur absence sur d'autres sites), en particulier pour le type I miniature. Mais quel(s) rôle(s)?

L'amphorette du type II (variantes $A$ et $B$ ) a une silhouette qui évoque, incontestablement, les petites amphores gauloises à fond plat ; celles-ci étaient vraisemblablement, dans une très large majorité de cas, sinon dans tous les cas, réservées aux vins de Narbonnaise (40). Sans autre preuve que cette assimilation par la forme, il y a de bonnes raisons de penser que ces petites amphores de Mandelieu étaient destinées au même usage (41). D'ailleurs, on l'a vu, certains détails dans la forme (que ce soit le fond, la panse ou les différents composants du col, ou les anses) s'inscrivent dans les conceptions morphologiques que l'on retrouve sur les autres amphores de la même famille, en Gaule méridionale. Le volume de 131 (une urna) ne s'oppose pas à un tel contenu.

L'amphorette du type I, en revanche, avec une forme pourtant bien particulière, non seulement parait inconnue dans les sites régionaux limitrophes, mais ne semble pas, non plus, trouver de similitudes avec des amphorettes conçues dans d'autres régions. Ce petit conteneur de 3,25 1 (un congius) apparait, dans le monde de la céramique connue,

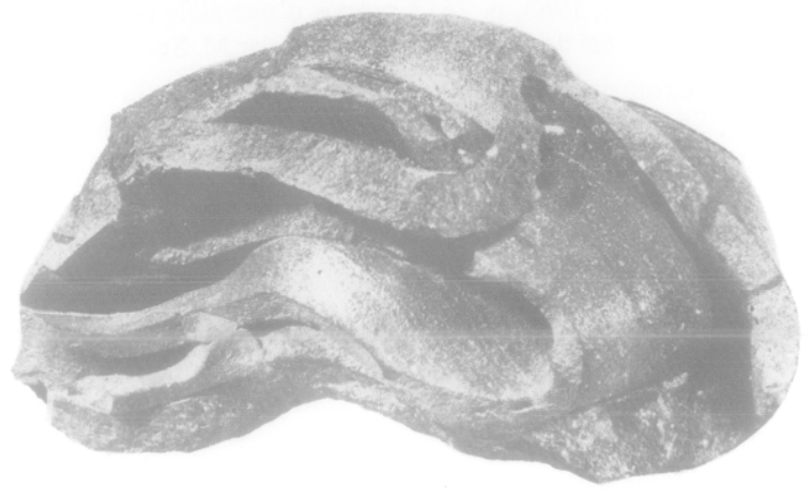

Fig. 17 - Rate de cuisson d'amphorette (cl. auteur). 
comme un hapax. De plus, sa très faible capacité en fait un objet tout à fait original pour lequel, en fonction de l'esprit des connaissances et des recherches contemporaines, il nous est pratiquement interdit de choisir un usage parmi les différents produits traditionnels transportés ou stockés dans les amphores: ni le vin, ni l'huile, ni la saumure. Alors quel produit ? On l'ignore, même si on est tenté de chercher parmi des substances rares ou précieuses, sans doute liquides, peut-être un condiment (42).

\section{Conclusion}

Il parait plausible de considérer que la production des amphorettes justifie pour une large part l'atelier, que leur fabrication est à l'origine de la création de celui-ci. Il semble évident, également, que ces conteneurs répondent à des besoins agricoles et, peut-être, piscicoles. Mais s'agit-il de ceux de cette villa, uniquement, ou de ceux de plusieurs villae? La localisation de l'atelier permettrait, sans doute, de préciser la réponse. Si l'atelier se trouve sur la rive gauche de l'Argentière, comme nous l'avons supposé, il peut se situer sur le territoire de la villa. En revanche, s'il est à placer vers la Siagne, il est possible qu'il soit en contact avec plusieurs villae ; à moins qu'il ne fasse partie d'un quartier artisanal dans la mouvance d'ad Horrea, vers l'Argentière ou vers la Siagne, selon que l'on assimile cette station à La Napoule ou à Mandelieu.

On constate, enfin, que cet atelier ne semble pas produire longtemps ; les contextes stratigraphiques, en tout cas, ne permettent pas d'être précis sur ce plan, mais la période couverte pourrait ne pas excéder une ou deux générations. On entrevoit les implications sur l'économie de la villa. L'émergence de celle-ci n'est pas accompagnée par la création de l'atelier : une période de vingt ou trente ans les en sépare et, ultérieurement, la disparition de l'atelier ne produit pas d'effets discernables, à la fouille, dans les bâtiments du domaine mis au jour. Faut-il s'en étonner?

\section{NOTES}

* C.N.R.S., Centre Camille Jullian, Université de Provence - 13621 AIX-EN-PROVENCE

1 - La fouille a été co-dirigée par L. Aygueparse, M. Fixot et Y. Codou qui nous ont confié l'étude du matériel céramique (environ 60000 tessons) ; nous les remercions bien vivement. L'étude de ce matériel est en cours.

2 - Le thème du présent article a fait l'objet d'une communication au Congrès de la S.F.E.C.A.G. (Fréjus, 1984).

3 - Le site n'est pas inconnu des médiévistes puisqu'il est mentionné dans des textes du début du XIème s. pour un prieuré de l'abbaye de Lérins, sous le vocable de Sainte-Marie. A la fin du Moyen-Age, une nécropole de tombes à coffrage s'installe sur les ruines de la villa.

4 - Les lits de ces cours d'eau, dans cette région de basses terres littorales, se sont sans doute déplacés sensiblement au cours des temps ; celui de l'Argentière, limité au sud par des reliefs, pourrait éventuellement avoir été plus proche du site dans l'Antiquité.

5 - Avec, au moins, des réserves à sous-entendre quant au terme, on localise depuis peu une aire de production d'amphores à pâte "marseillaise" entre les basses vallées de la Siagne et de l'Argentière (cf. J.-Cl. Echallier, La provenance des amphores massaliètes, dans D.A.M., 5,1982, p. 142, où l'auteur décrit ainsi l'argile : "pâte céramique monogénique... dégraissée à l' aide d'une arène issue d'un filon de gneiss granitoïde pegmatitique à muscovite et tourmaline") ; cette zone dépressionnaire et argileuse étant géographiquement assez limitée (3 ou $4 \mathrm{~km} \mathrm{2)}$, il y a tout lieu d'imaginer que les céramiques qui font l'objet de cet article sont réalisées, sinon dans une pâte qui correspond à cette définition, du moins dans une pâte analogue. L'argile présente sur le site est directement utilisable pour confectionner des vases comme l'a démontré une expérience menée pendant la fouille.

6 - Au nord de Mandelieu, la villa du Château de la Tour est mentionnée par F. Benoit (Recherches sur l'hellénisation du Midi de la Gaule, Aix-en-Provence, 1965, p. 24) à propos d'une stèle portant une inscription grecque. A l'est de Mandelieu, la villa proche de la colline Saint-Cassien a été demièrement observée par M. Sechter (communication au Congès de la S.F.E.C.A.G., Fréjus, 1984, inédit).

7 - La voie romaine franchit la Siagne à proximité de la station ad Horrea que l'on peut assimiler à La Napoule (?) (cf. R. Boyer et P.-A. Février, Stations routières romaines de Provence, dans R.S.L., 25, 1959, p. 162-168, ainsi que G. Barruol, Les peuples pré-romains du sud-est de la Gaule, étude de géographie historique, suppl. 1 à la R.AN., Paris, 1969, p. 73).

8 - L. Aygueparse, M. Fixot et Y. Codou, Un temple de Mithra à Mandelieu, dans Les dossiers d'Hist. et Archéol., 57, 1981, p. 85-86. Sur la datation de cet abandon, $\mathrm{Cl}$. Brénot, Les dépôts monétaires votifs des fouilles de Mandelieu, dans Bull. de la Soc. Nat. des Ant. de France, 1983, p. 275-277.

9 - On compte 351 fragments surcuits de céramique ; ne sont pas décomptées quelques dizaines de coulures ou scories.

10 - On indiquera dans le catalogue les tessons qui proviennent de ces couches profondes, en précisant le contexte chronologique. L'étude de l'abondant matériel livré par cette villa est en cours ; il est donc possible qu'au terme de cette recherche la datation avancée ici subisse une correction.

11 - On n'envisagera pas l'éventuelle production d'amphores de types classiques ; la fouille n'a d'ailleurs pas fourni de surcuits ; seule la qualité des pâtes suggère une production "locale" pour les formes Dressel $2 / 4$ et Gauloise 4 et 5. 
12 - Rappelons que la couleur grise est réalisable si le four est hermétiquement clos durant la post-cuisson, et que la couleur brune découle d'un colmatage imparfait qui laisse entrer de l'air (cf. M. Picon, Introduction à l'étude technique des sigillées de Lezoux, dans Centre de Rech. sur les Techn. Gréco-Rom., Dijon, 2, 1973, p. 55-75).

13 - Pour le premier bord, on se limitera à renvoyer à Chr. Goudineau, Note sur la céramique commune grise gallo-romaine de Vaison, dans RA.N., X, 1977, p. 153-169, fig. 2, forme 2 c ; pour le second bord, A. Desbat, C. Laroche et E. Merigoux, Note préliminaire sur la céramique de la rue des Farges à Lyon, dans Figlina, 4, 1979, p. 1-17, fig. VII, ${ }^{\circ} 2$.

14 - Des jattes, de morphologie non pas identique mais équivalente, sont bien connues en Provence occidentale ; caractéristiques du Ier $\mathrm{s}$. av. n. è., elles sont encore largement fabriquées et utilisées pendant l'époque augustéenne (cf. P. Arcelin, La céramique modelée au ler $s$. avant J.-C. dans les Bouches-du-Rhône, thèse de IIIème cycle, Aix-en-Provence, 1979, dactyl. : voir les variantes 2 et 5 de la forme $4 \mathrm{~d}$; le bord rentrant et l'adjonction des préhensions pourraient trahir des influences italiques).

15 - On a exclu les fragments qui pourraient appartenir à des productions en pâte sombre, de même que ceux qui se distinguent difficilement des amphorettes.

16 - Le premier col autorise une comparaison avec des cruches produites en pâte sombre (cf. A. Desbat, C. Laroche et E. Merigoux, Note préliminaire...., l.c., fig. VI, $\left.\mathrm{n}^{\circ} 4\right)$ pour l'époque flavienne, ou avec des cruches en pâte claire, comme à Vindonissa (cf. E. Ettlinger et C. Simonett, Römische Keramik aus dem Schutthügel von Vindonissa, Veröffentlichungen der Gesellschaft Pro Vindonissa, 3, Bâle, 1952. fig. 24, $n^{\circ} 541$ ) pour le Ier s. de $n$. è. Le deuxième fragment est à rapprocher d'une cruche à deux anses découverte à Camulodunum (cf. J. P. Gillam, Types of roman coarse pottery in Northern Britain, dans Archeologia Aeliana, 4ème série, XXXV, 1957, type $3 \mathrm{c}, \mathrm{n}^{\circ} 169$, datant du milieu du Ier s. de n. è.) ct d'une cruche à anse de Vindonissa (cf. E. Ettlinger et C. Simonett, Römische Keramik..., l.c., fig. 19, $\mathrm{n}^{\circ} 430$ ).

17 - Ces mortiers, de conception typiquement méditerranéenne, se rencontrent abondamment dans les habitats gallo-romains ; pour une comparaison avec un site régional, N. Lamboglia, Gli scavi di Albintimilium e la cronologia della ceramica romana, Bordighera, 1950, fig. $63, \mathrm{n}^{\circ} 44$, pour l'époque flavienne.

18 - Là encore les références seraient fort nombeuses; dans la région, et pour le milieu du Ier s. de n. è., cf. A. Olivier et G. Rogers, Le monument de Vaugrenier (Alpes-Maritimes), dans RAN., XI, 1978, fig. 44, $\mathrm{n}^{\circ} 2$, ainsi que N. Lamboglia, Gli scavi..., o. c., fig. 16, $\mathrm{n}^{\circ} 63$, pour l'époque flavienne.

19 - Aucun exemplaire entier ; la mesure est celle qui découle de la restitution proposée sur la fig. $10, n^{\circ} 32$.

20 - Le nombre de fragments qui se rapportent aux amphorettes, tessons avec forme et tessons sans forme, du type I et des autres types, est important en quantité : env. 17900 fragments, et en proportion de l'ensemble du matériel céramique : près de $30 \%$. Parmi les éléments avec forme, on a pu dénombrer 1315 fragments appartenant au type $I$.

21 - On aura remarqué que dans certains cas les anses, parce que trop courtes, laissent difficilement passer les doigts.

22 - Le calcul a été réalisé sur la seule amphorette restituée graphiquement, selon la méthode de Y. Rigoir (Méthode géométrique simple de calcul du volume des contenants céramiques, dans D.A.M., 4, 1981, p. 193-194); on a arrêté le volume au tiers inférieur du col. Le poids approximatif est de $3,5 \mathrm{~kg}$.

23 - L'amphorette mesurée est celle qui présente le plus fort diamètre.

24 - Nous n'avons pas, cependant, vu le matériel de l'Ile Sainte-Marguerite, ni celui de Peymeinade. Pour cette forme, on ose à peine écrire qu'il y a quelques vagues ressemblances avec la forme Dressel $2 / 4$... Cet objet sera répertorié en G. 11 dans un article à paraitre de F. Laubenheimer, Production et chronologie des amphores gauloises : une nouvelle problématique, dans Anfore romane e storia economica : un decennio di ricerche, Siena, 1986.

25 - Voir la note 20. Parmi les éléments de forme, on a pu dénombrer 1064 fragments appartenant au type II (variantes $A$ et $B$ ).

26 - Aucun exemplaire entier ; la mesure est celle qui découle de la restitution proposée sur la fig. $11, n^{\circ} 38$.

27 - Voir la note 22 ; mêmes remarques. Le poids approximatif est de $5 \mathrm{~kg}$.

28 - P.-Y. Genty, J.-L. Fiches, L'atelier de potiers gallo-romains d'Aspiran (Hérault), dans Figlina, 3, 1978, fig. 2, $\mathrm{n}^{\circ} 2$, pour la fin de l'époque augustéennc.

29 - A. Tchemia, J.-P. Villa, Note sur le matériel recueilli dans la fouille d' un atelier d'amphores à Velaux (Bouches-du-Rhône), dans Méthodes classiques et méthodes formelles dans l'étude des amphores, Rome, 1977, fig. 6, pour les amphores du "dépotoir a", datables, sans plus de précisions, du Ier $\mathrm{s}$. de $\mathrm{n}$. è.

30 - F. Laubenheimer, La production des amphores en Gaule Narbonnaise, Ann. Litt. Univ. de Besançon, 327, Paris, 1985 , p. $302-306$. 31 - Aucun exemplaire entier ; la mesure est celle qui découle de la restitution proposée sur la fig. $12, n^{\circ} 43$.

32 - Voir la note 22 ; mêmes remarques.

33 - A. Tchernia, J.-P. Villa, Note sur le matériel..., l.c., fig. 7, $\mathrm{n}^{\circ} 2$.

34 - On ne dispose que d'une photographie (cf. F. Laubenheimer, La production..., o. c., p.306 et fig. 164, p. 305) que l' auteur attribue à la forme Gauloise 7, alors que le col, nettement en diabolo, s'en distingue radicalement. Cette petite amphore de Mandelieu sera placée dans la forme G. 2 (tradition des amphores de Marseille) par F. Laubenheimer, Production et chronologie..., l. c. (à paraittre).

35 - Cet ateliet n'est pas une création isolée. A $8 \mathrm{~km}$ vers l'est, dans le centre de Cannes, trois fours et une décharge d'amphores de la forme Gauloise 6 ont été observés, en 1978, par M. Sechter (contexte Ier s. de n. è. ?) (cf. F. Laubenheimer, La production..., o. c., p. $95-$ 97). Par ailleurs, la villa proche de la colline Saint-Cassien a livré, dans des tranchées réalisées en 1979 à l'intérieur d'un complexe sportif, des ratés de cuisson d'amphores (communication de M. Sechter au Congrès de la S.F.E.C.A.G., Fréjus, 1984, inédit).

36 - Sur ces disques, voir en dernier lieu F. Laubenheimer, K. Gruel, A. Naciri, M. Pasquier et F. Widemann, L'atelier de potiers galloromains de Puyloubier (Bouches-du-Rhône), dans D A.M., 7, 1984, p. 97-110, et plus spécialement p. 103-104 et fig. 11, $\mathrm{n}^{\circ} 1-2$. Durant l'automne et l'hiver 1984-85, nous en avons trouvé une série de seize dans les fouilles de l'Archevêché à Aix-en-Provence : huit sont identiques par la taille et la facture (diam. ext. entre 9 et $10 \mathrm{~cm}$, diam. de l'ouv. centrale entre 2,3 et 2,6 cm), les autres étant plus grands (diam. 
ext. jusqu'à $15 \mathrm{~cm}$, diam. de l'ouv. centrale jusqu'à $4 \mathrm{~cm}$ ) (cf. M. Fixot, J. Guyon, J.-P. Pelletier et L. Rivet, Les fouilles de la cour de l'Archevêché, dans Doc. d'Archéol. Aixoise, 1, 1985, fig. 53). Le site a livré une série de vases voilés et mécuits qui témoignent de la proximité d'un atelier de potiers.

37 - F. Hermet, La Graufesenque, Paris, 1934, p. 275 et pl. 123, n 3, défini comme "cylindre énigmatique"; les dimensions sont bien supérieure (diam. : $24,8 / 28,6 \mathrm{~cm} ; \mathrm{h}: 18 \mathrm{~cm}$ ).

38 - Disons-le, l'exemplaire $n^{\circ} 59$ nous a fait penser à un col d'amphorette et le n ${ }^{\circ} 55$ à un col de cruche ; on sait que ceux-ci sont tournés à part. L'hypothèse est totalement balayée par les autres colliers qui, eux, n'ont aucun point commun avec ces éventuels cols.

39 - Le potier n'a-t-il pas dans les yeux la silhouette d'un cratère à volutes?

40 - B. Liou, R. Marichal, Les inscriptions peintes sur amphores de l' anse Saint-Gervais à Fos-sur-mer, dans Archaeonautica, 2, 1978, p. 109-181, plus spécialement p. 145-159, 175-176 et 179-181; en dernier lieu, F. Laubenheimer, La production..., o. c., p. 399-406. Les amphores G. 1, G. 5 et G. 7 présentent deux modules : l'un de 301 env. et l'autre de $13 / 14$ l; 14,25 l pour la G. 1 de Lattes (ibid., p. 254 et fig. 112), 13 l env. pour la G. 5 de Fréjus (ibid., p. 299 et fig. 158), la G. 7 n'ayant pu être mesurée (ibid., p. 302-307). Les amphorettes du type II de Mandelieu s'inscrivent donc bien dans cette série des "demi-amphores".

41 - Les amphores à vin sont systématiquement poissées; sur la plupart des tessons récupérés dans des sites terrestres, l'absence de poix est un phénomène général, le milieu de conservation ayant la propriété de faire disparaître cet enduit.

42 - Compte-tenu de la proximité du rivage, il est possible de penser au garum ou à l'un de ses dérivés, mais on manque du moindre indice pour étayer cette hypothèse. 